\title{
Mechanistic Insights and Implications of Dearomative Rearrangement in Copper-Free Sonogashira Cross- Coupling Catalyzed by Pd-Cy*Phine
}

Adrian M. Mak ${ }^{a}$, Yee Hwee Lim ${ }^{b}$, Howard Jong ${ }^{b}$, Yong Yang ${ }^{b}$, Charles W. Johannes $^{b}$, Edward G. Robins ${ }^{c}$, Michael B. Sullivan ${ }^{a}$

anstitute of High Performance Computing, 1 Fusionopolis Way, \#16-16 Connexis, Singapore 138632

${ }^{\mathrm{b}}$ Institute of Chemical and Engineering Sciences, 8 Biomedical Grove, Neuros \#07-01, Singapore 138665

'Singapore Bioimaging Consortium, 11 Biopolis Way, Helios \#02-02, Singapore 138667

Supplementary Information

\section{Contents}

1. Free energy profiles of Sonogashira Cross-coupling S2 catalytic cycle for $\mathrm{R}=\mathrm{Ph}, \mathrm{H}, \mathrm{Me}, \mathrm{OMe}, \mathrm{F}, \mathrm{CN}$

2. Intrinsic Reaction Coordinate Analysis for Transition States S9 
1. Free energy profiles of Sonogashira Cross-coupling catalytic cycle for $\mathrm{R}=\mathrm{Ph}, \mathrm{H}, \mathrm{Me}, \mathrm{OMe}, \mathrm{F}, \mathrm{CN}$

$\mathrm{R}=\mathrm{Ph}$

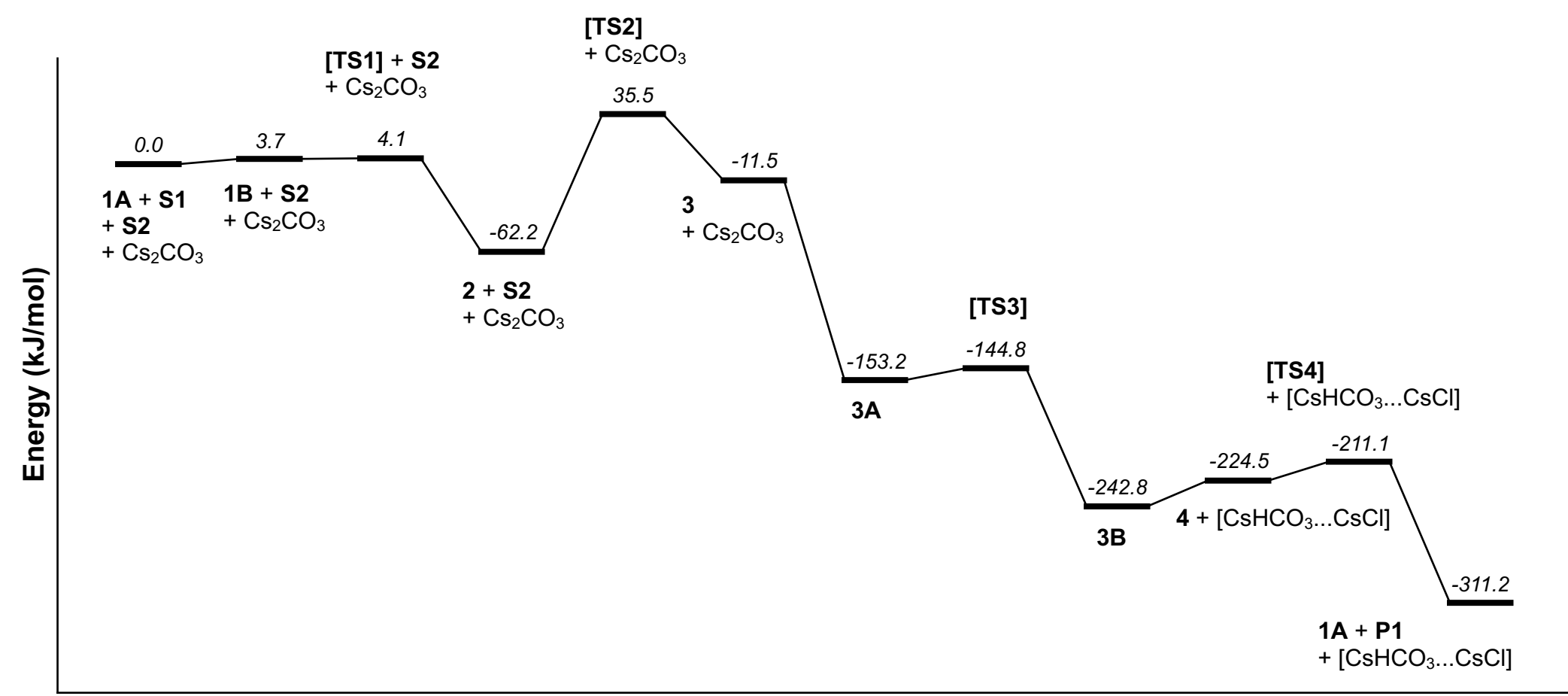

Reaction Progress 
Figure S1. Schematic free energy profile of Sonogashira cross-coupling for $\mathrm{R}=\mathrm{Ph}$. 
$\mathrm{R}=\mathrm{H}$

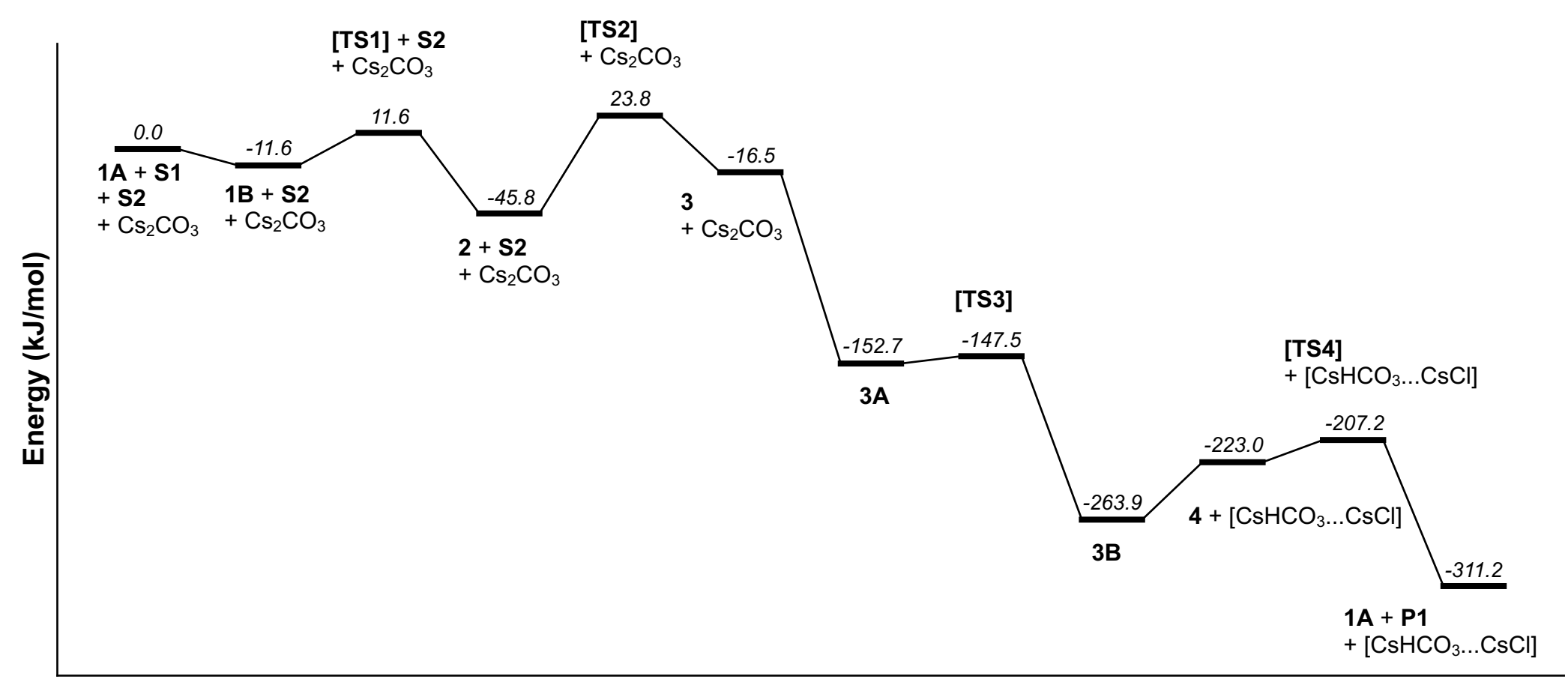

Reaction Progress

Figure S2. Schematic free energy profile of Sonogashira cross-coupling for R $=\mathrm{H}$. 
$\mathrm{R}=\mathrm{Me}$

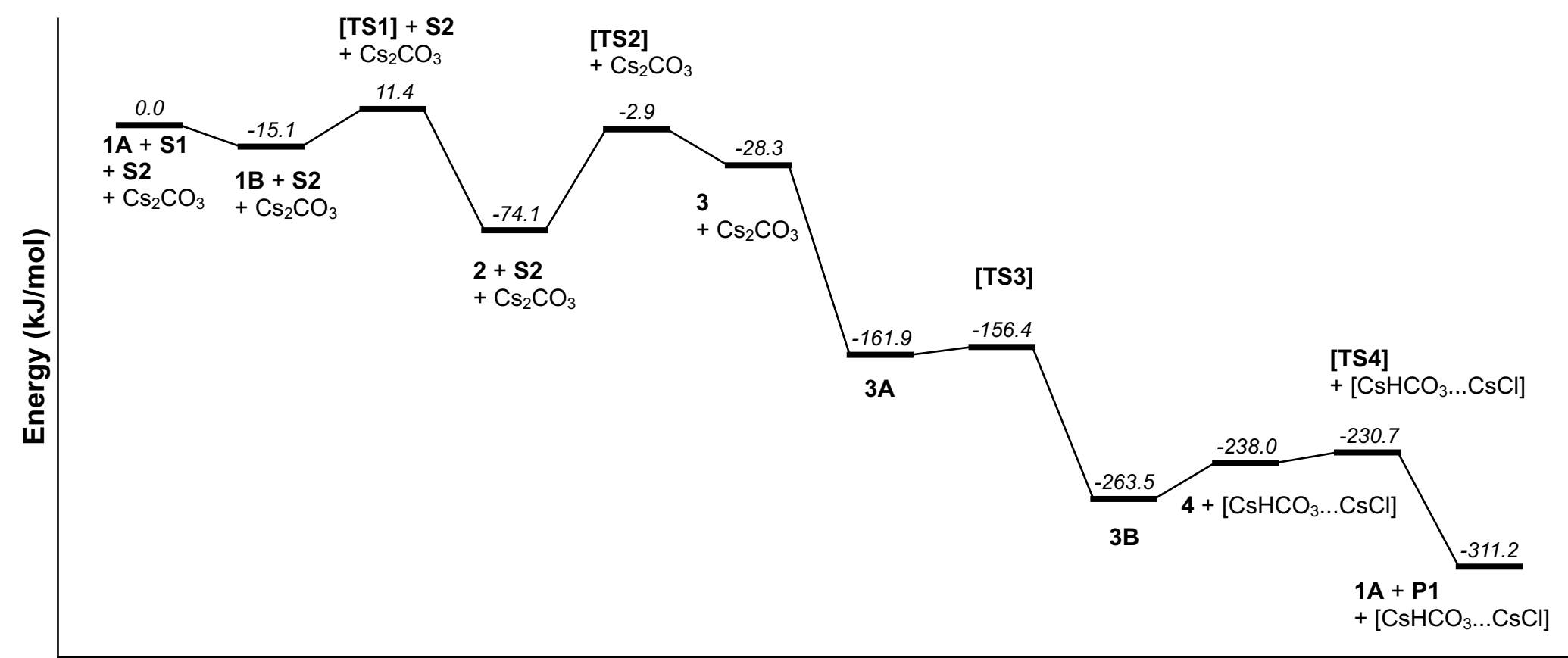

Reaction Progress

Figure S3. Schematic free energy profile of Sonogashira cross-coupling for $R=$ Me. 
$\mathrm{R}=\mathrm{OMe}$

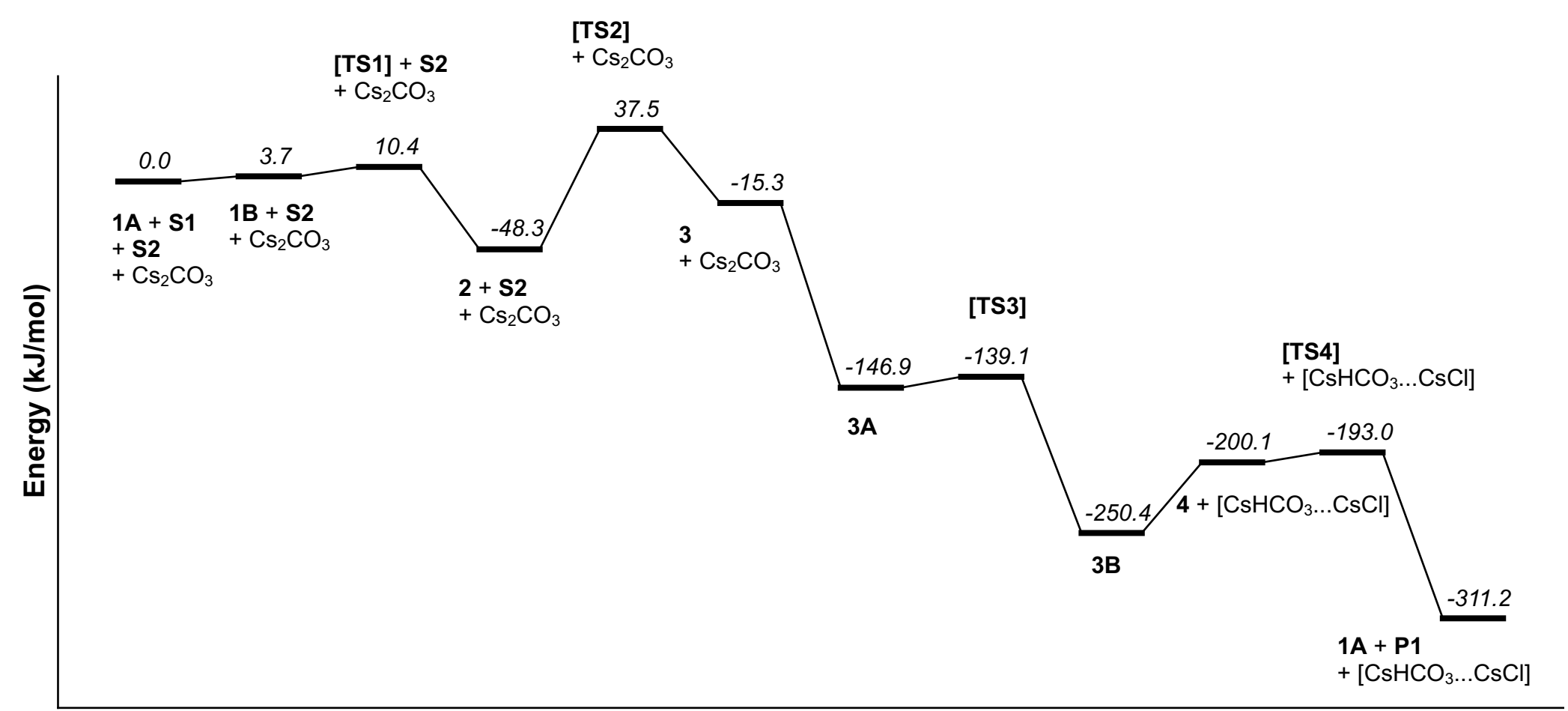

Reaction Progress

Figure S4. Schematic free energy profile of Sonogashira cross-coupling for $\mathrm{R}=\mathrm{OMe}$. 
$\mathrm{R}=\mathrm{F}$

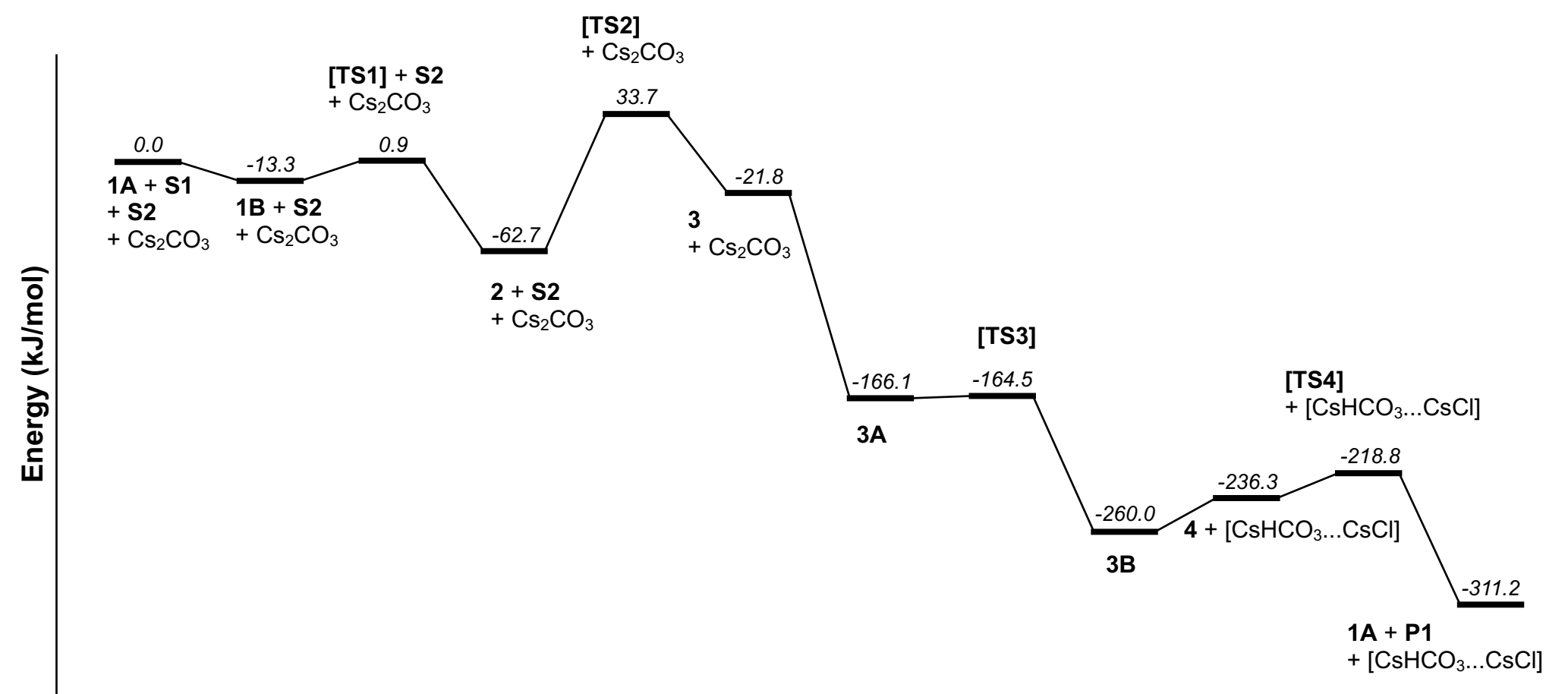

Reaction Progress

Figure S5. Schematic free energy profile of Sonogashira cross-coupling for $R=F$. 


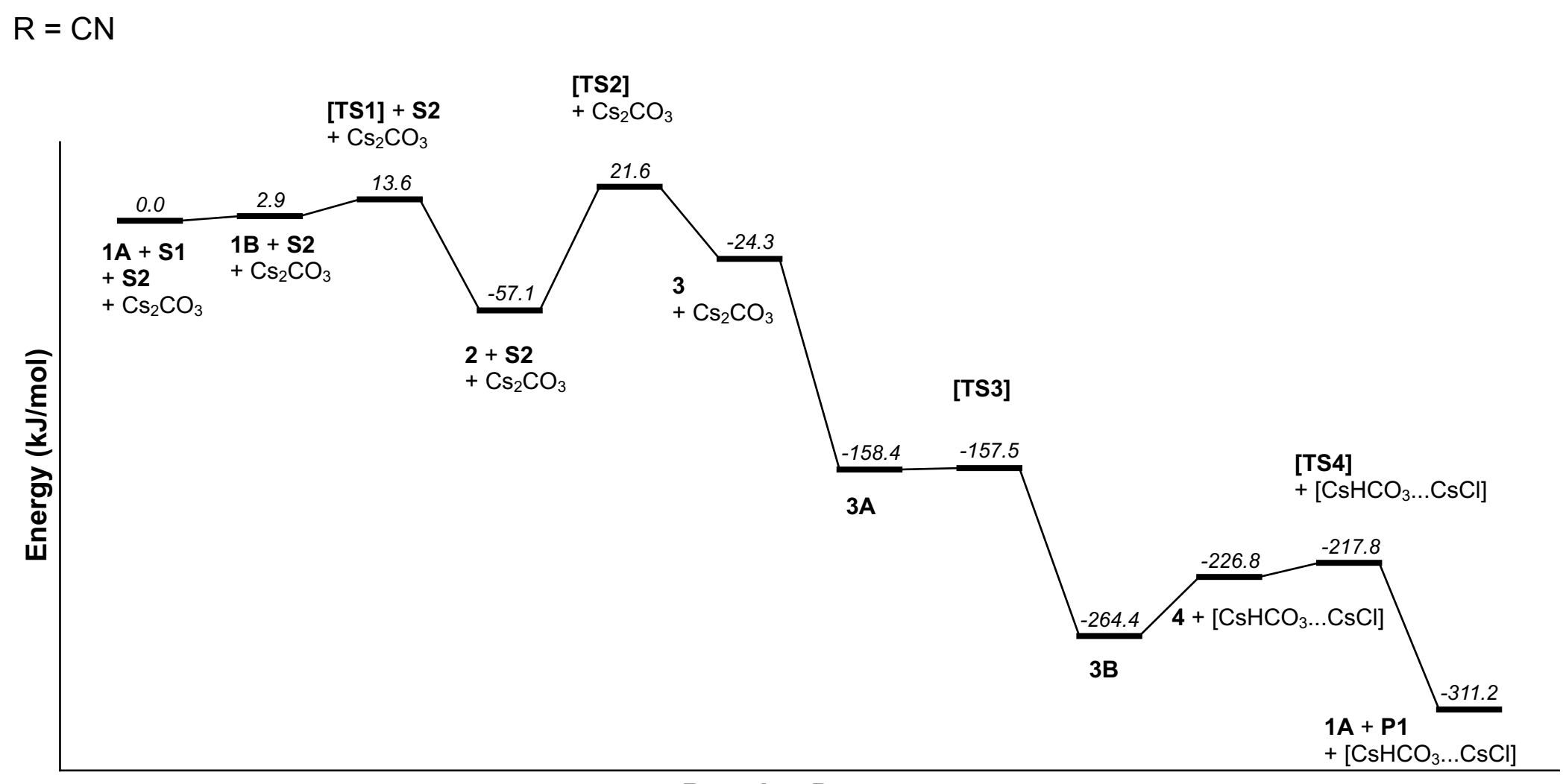

Reaction Progress

Figure S6. Schematic free energy profile of Sonogashira cross-coupling for $\mathrm{R}=\mathrm{CN}$. 
2. Intrinsic Reaction Coordinate Analysis for Transition States in Pd-Cy*Phine catalyzed Sonogashira cross coupling

\section{Method}

Transition states along the reaction pathway for the $\mathrm{Pd}-\mathrm{Cy}^{*}$ Phine were analyzed along their intrinsic reaction coordinate (IRC). The mass-weighted Hessian matrix obtained when computing harmonic frequencies is diagonalized, yielding one and only one negative eigenvalue. The normalized eigenvector $\mathbf{s}$ that corresponds to this negative eigenvalue (and imaginary frequency) is followed by taking small steepest descent steps of finite length, in our case, for a large amount of steps (typically $>50$ ), to obtain a putative product structure. The same process is repeated for the reverse direction of the eigenvector, -s, to obtain a putative reactant structure. These structures were then compared to the product and reactant complexes to verify that the transition state indeed corresponds to the reaction in question.

Plots of relative energy against $s$ for the TS's involved in the Pd-Cy*Phine catalyzed Sonogashira reaction are shown below, along with putative product and reactant structures obtained. The graphs were also oriented so that the products correspond to positive $\mathbf{s}$ and reactants correspond to negative $\mathbf{s}$, as the IRC routine is unable to determine their signs a priori. 


\section{Reaction Paths}

TS1

Energy $=-2052.234635$ a.u.

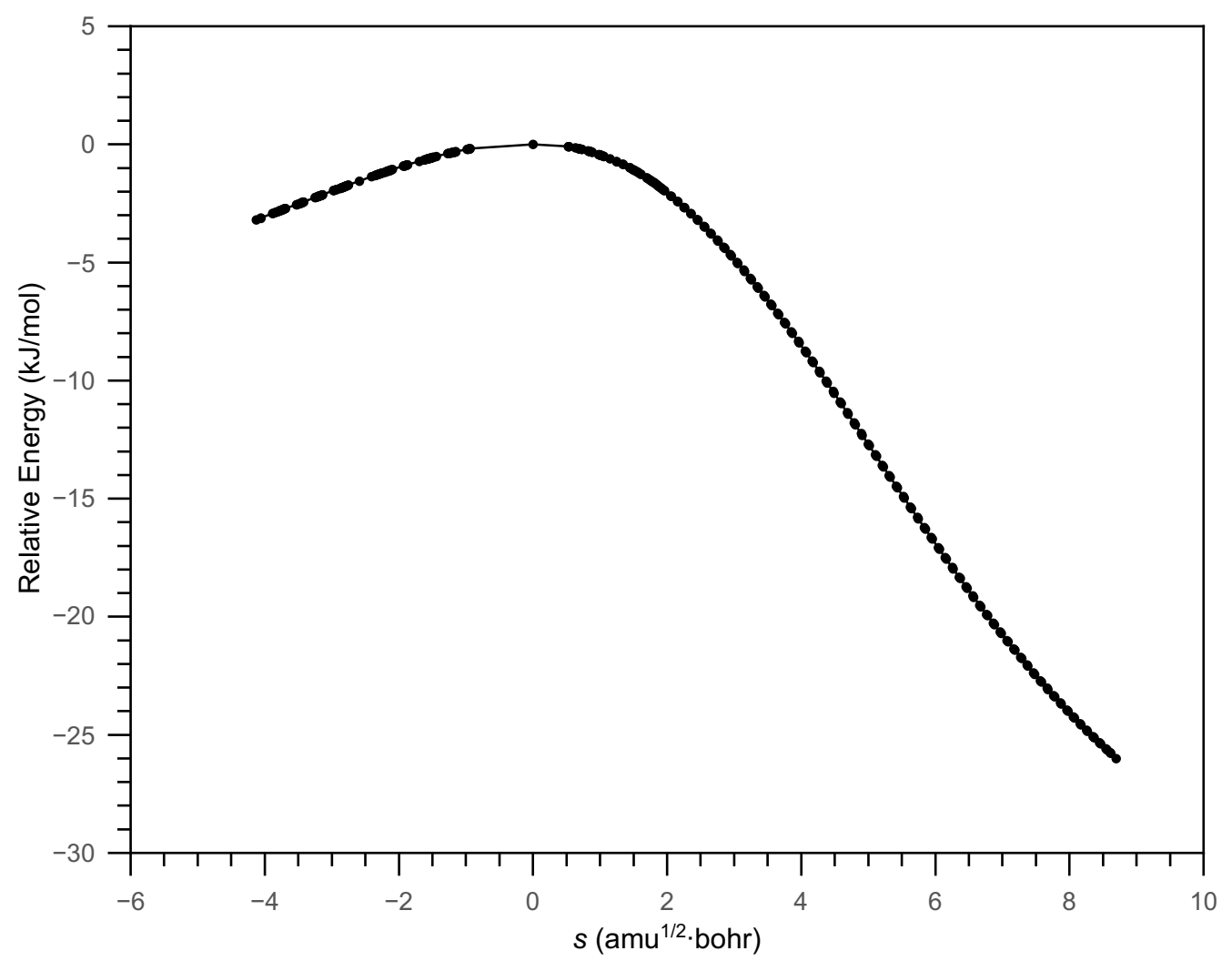

Figure S7. Reaction path following for transition state TS1, corresponding to the oxidative addition of $\mathbf{S 1}$ to $1 \mathrm{~B}$ to form 2. 


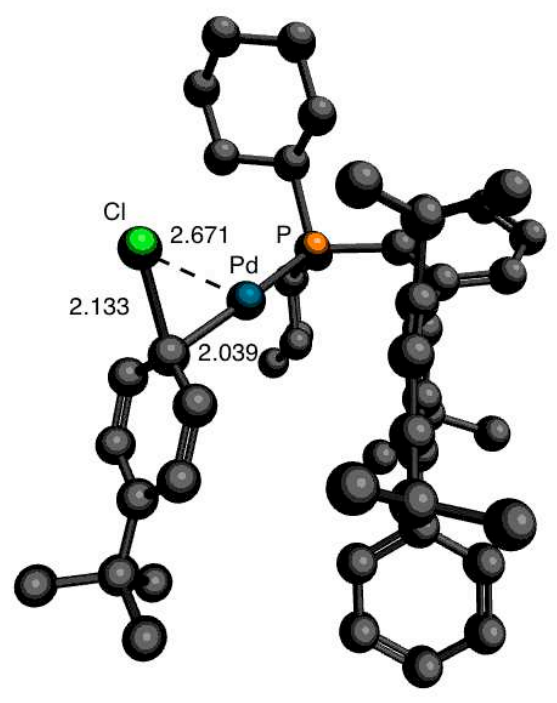

TS1

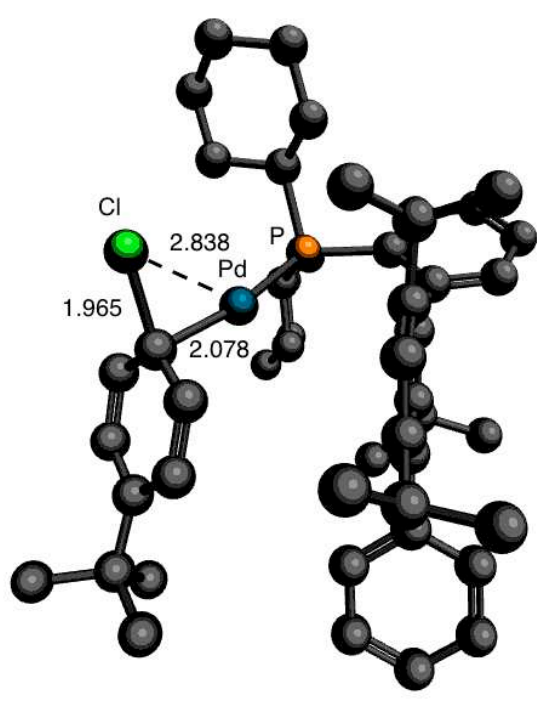<smiles>Clc1ccc(Br)cc1</smiles>

Reactant complex 1A
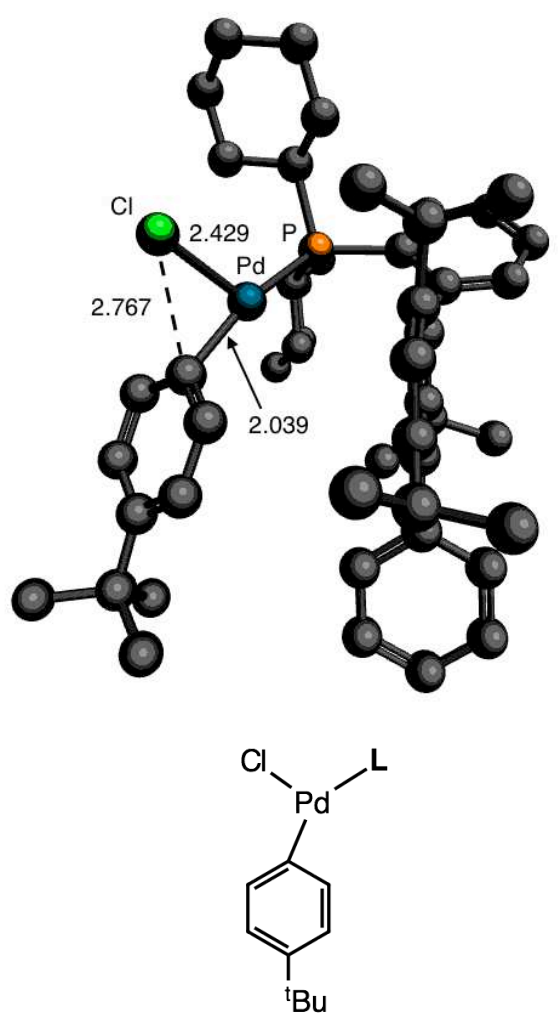

Product complex 2

Figure S8. Geometric structure of TS1 (top), and structures corresponding to the reactant (1B) and product (2) complexes on the intrinsic reaction path for oxidative addition. Interatomic distances are given in Ångstroms. 
TS2

Energy = -2360.183431 a.u.

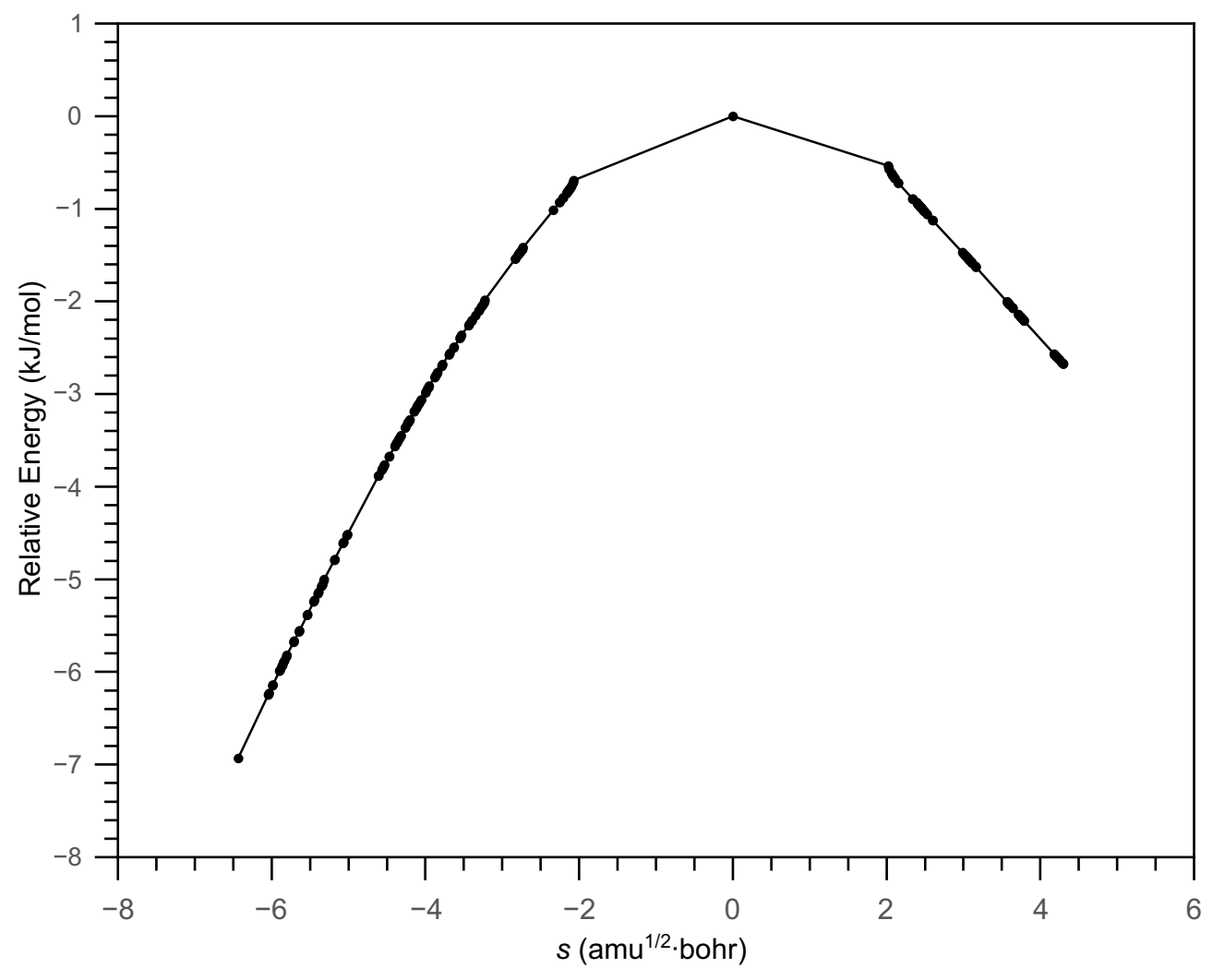

Figure S9. Reaction path following for transition state TS2, corresponding to the direct addition of alkyne $\mathbf{S} 2$ to the oxidative addition product $\mathbf{2}$ to yield $\mathbf{3}$. 


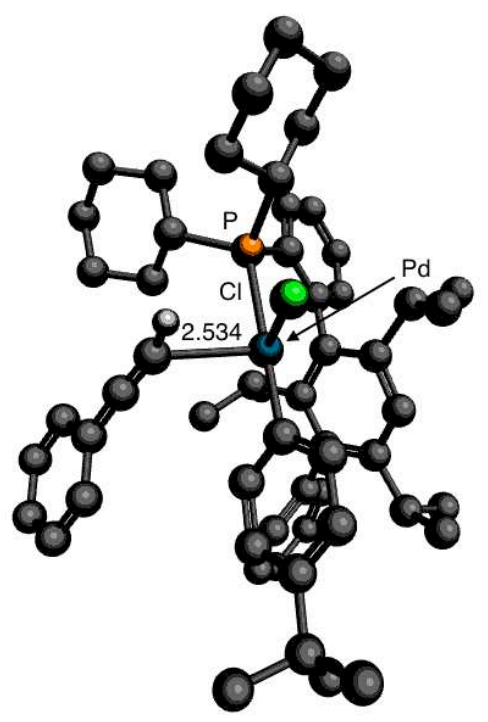

TS2
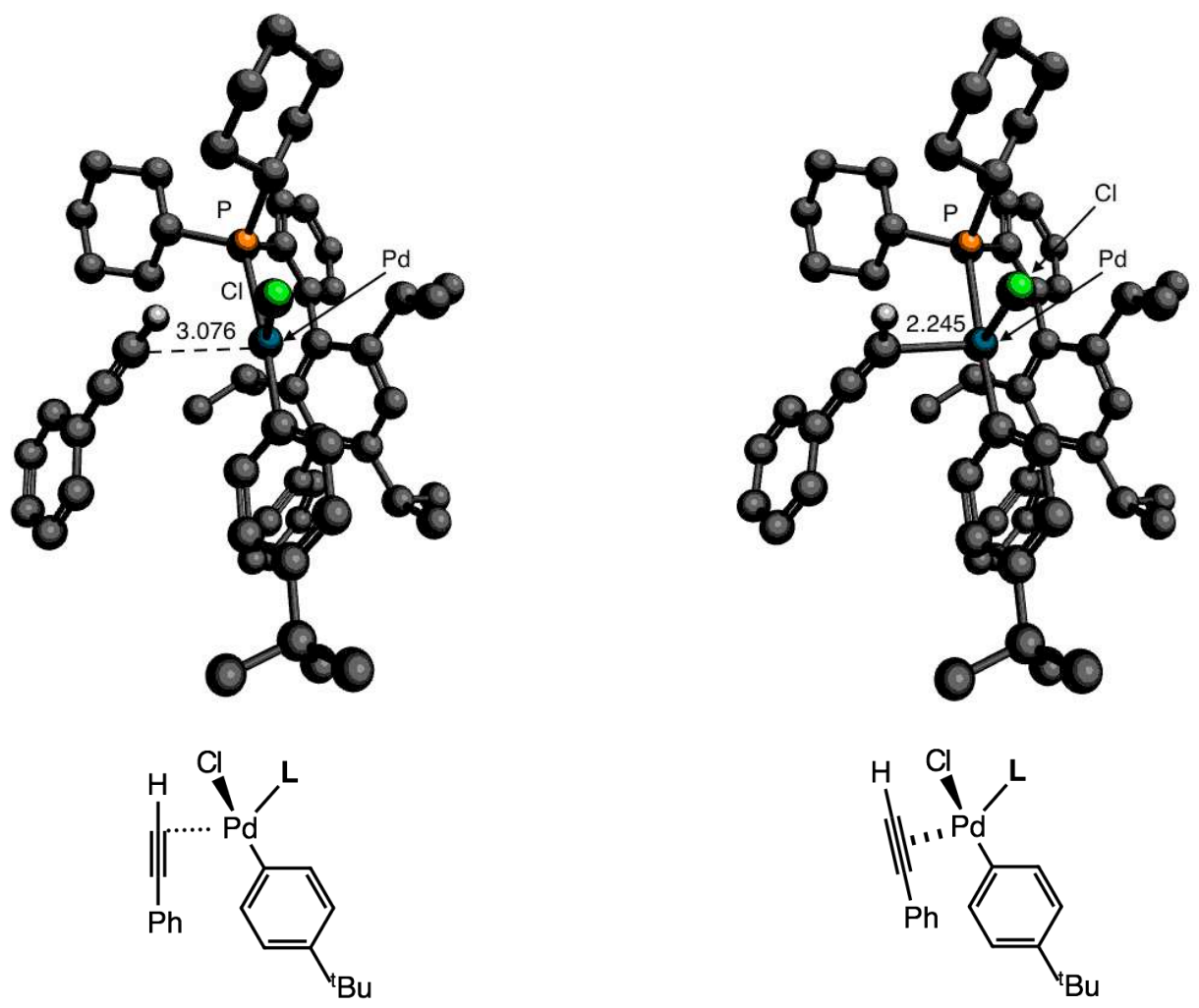

Reactant complex [2 + S2]

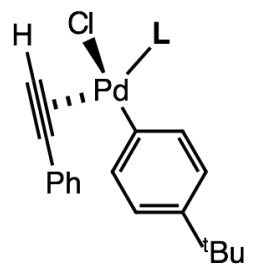

Product complex 3

Figure S10. Geometric structure of TS2 (top), and structures corresponding to the reactant $(2+\mathbf{S} 2)$ and product (3) complexes on the intrinsic reaction path for direct alkyne addition. Interatomic distances are given in Ångstroms. 
TS3

Energy $=-2663.552914$

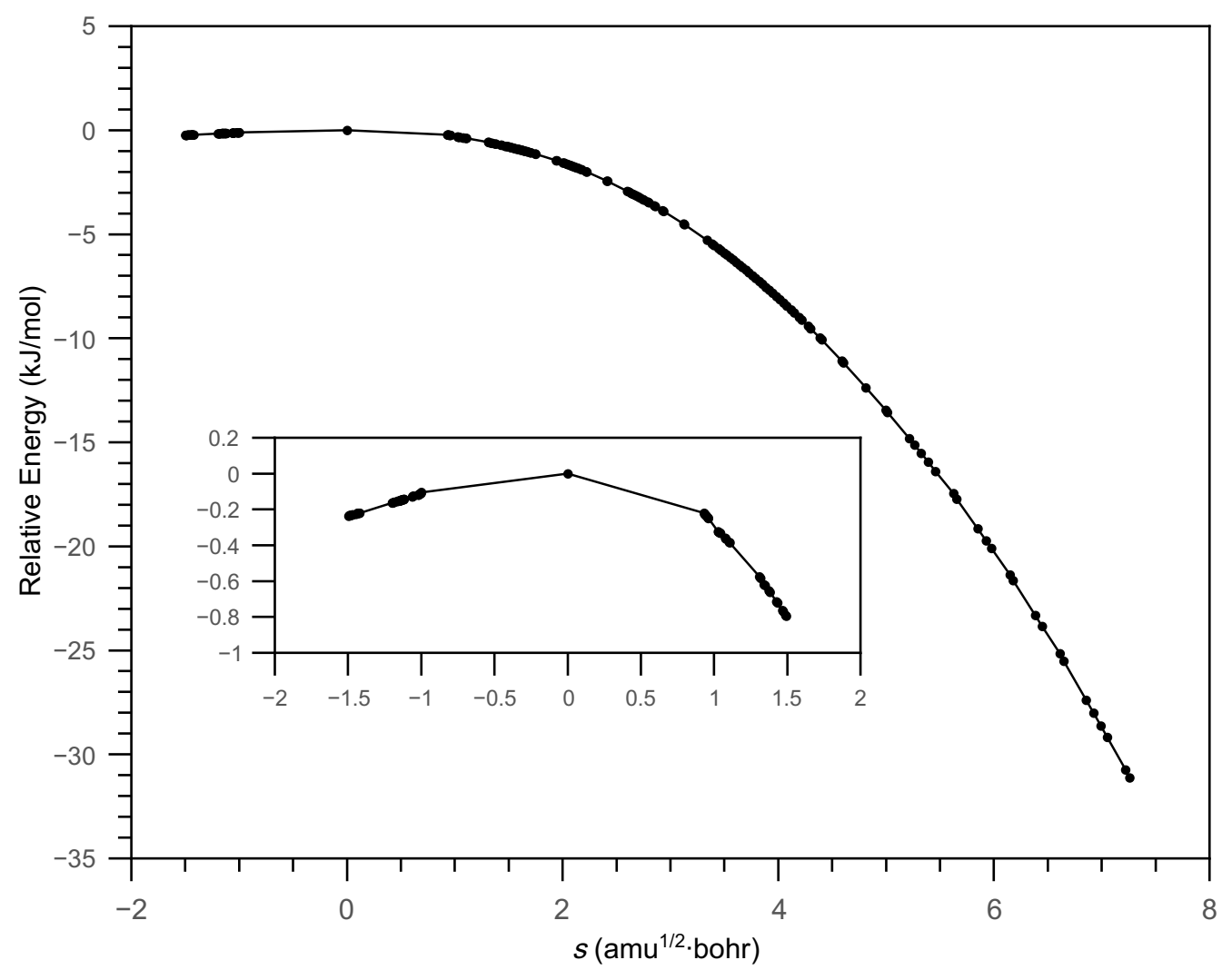

Figure S11. Reaction path following for transition state TS3, corresponding to the concerted deprotonation of alkyne $\mathbf{S} 2$ by $\mathrm{Cs}_{2} \mathrm{CO}_{3}$ and rearrangement of the alkynylide from $\pi$-type donor to $\sigma$-donor and $\pi^{*}$-acceptor to the central Pd in the reactant $3 \mathrm{~A}$ to yield the product $\mathbf{3 B}$. The inset shows the magnified intrinsic reaction path profile from $s=-1.5$ to $s=1.5$. 


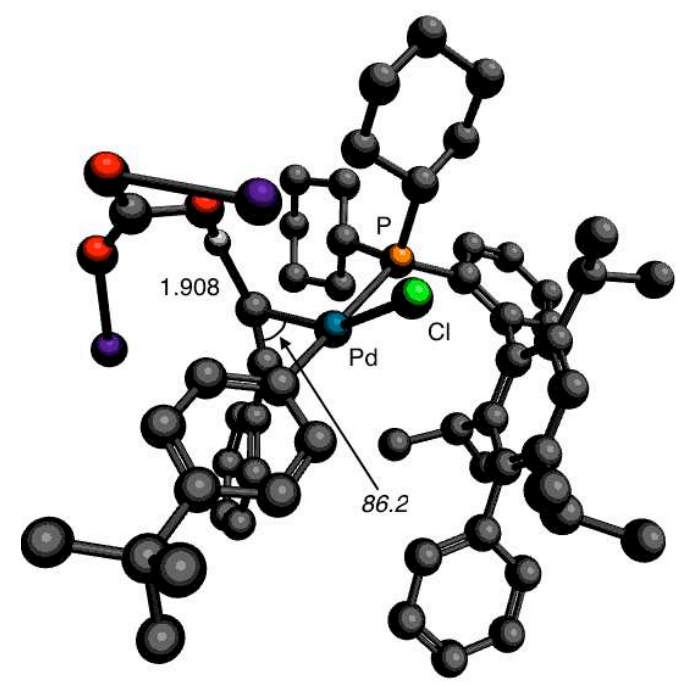

TS3
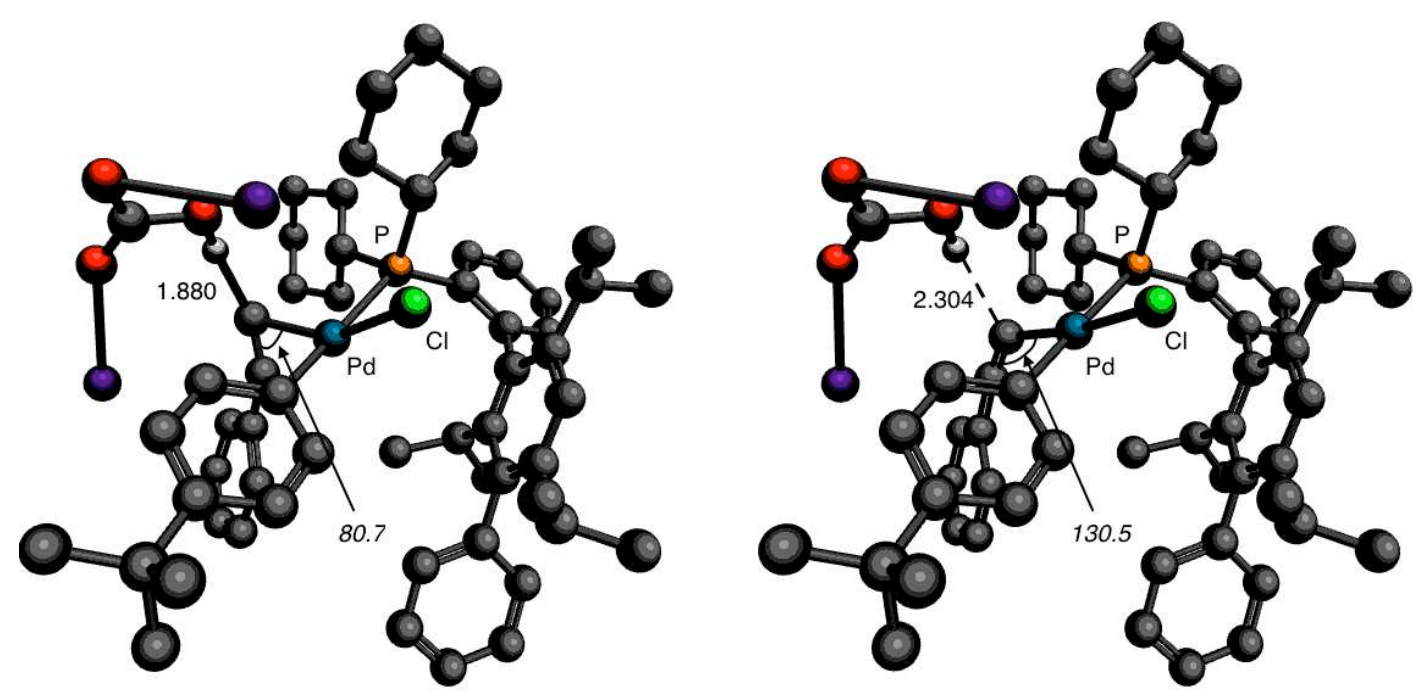

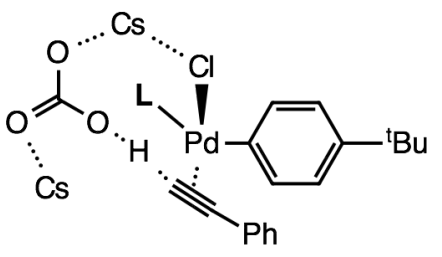

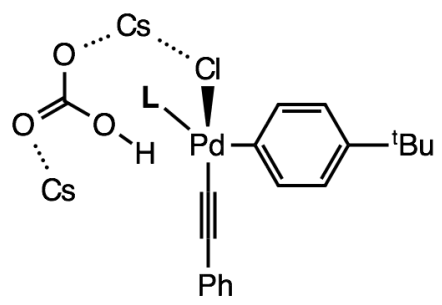

Reactant Complex

Product Complex

Figure S12. Geometric structure of TS3 (top), and structures corresponding to the reactant $(\mathbf{3 A})$ and product $(\mathbf{3 B})$ complexes on the intrinsic reaction path for concerted alkyne deprotonation and rearrangement. Interatomic distances are given in Ångstroms, bond angles are given in degrees and shown in italics. 
TS4

Energy $=-2344.622705$ a.u.

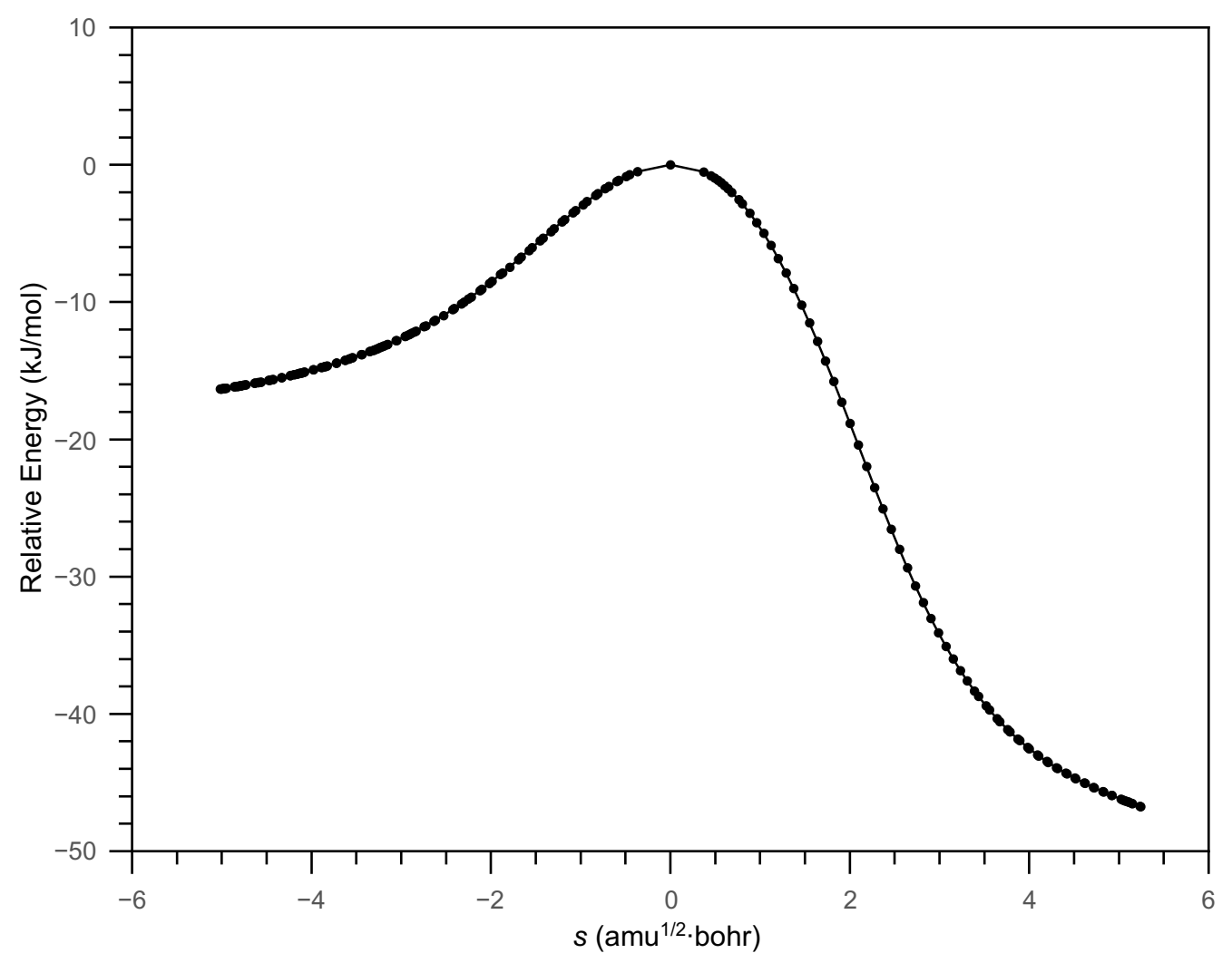

Figure S13. Reaction path following for transition state TS4, corresponding to the reductive elimination of $\mathbf{P} \mathbf{1}$ from $\mathbf{4}$ to form $\mathbf{1 A}+\mathbf{P} \mathbf{1}$. 


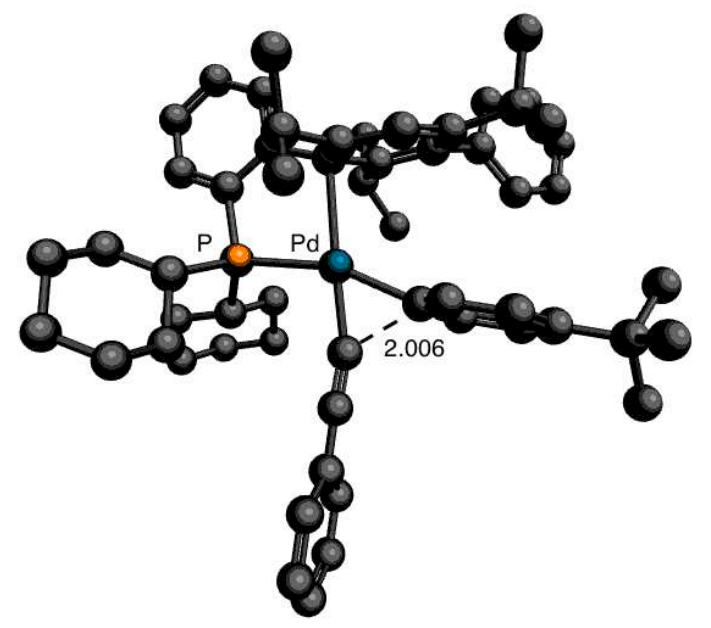

TS4
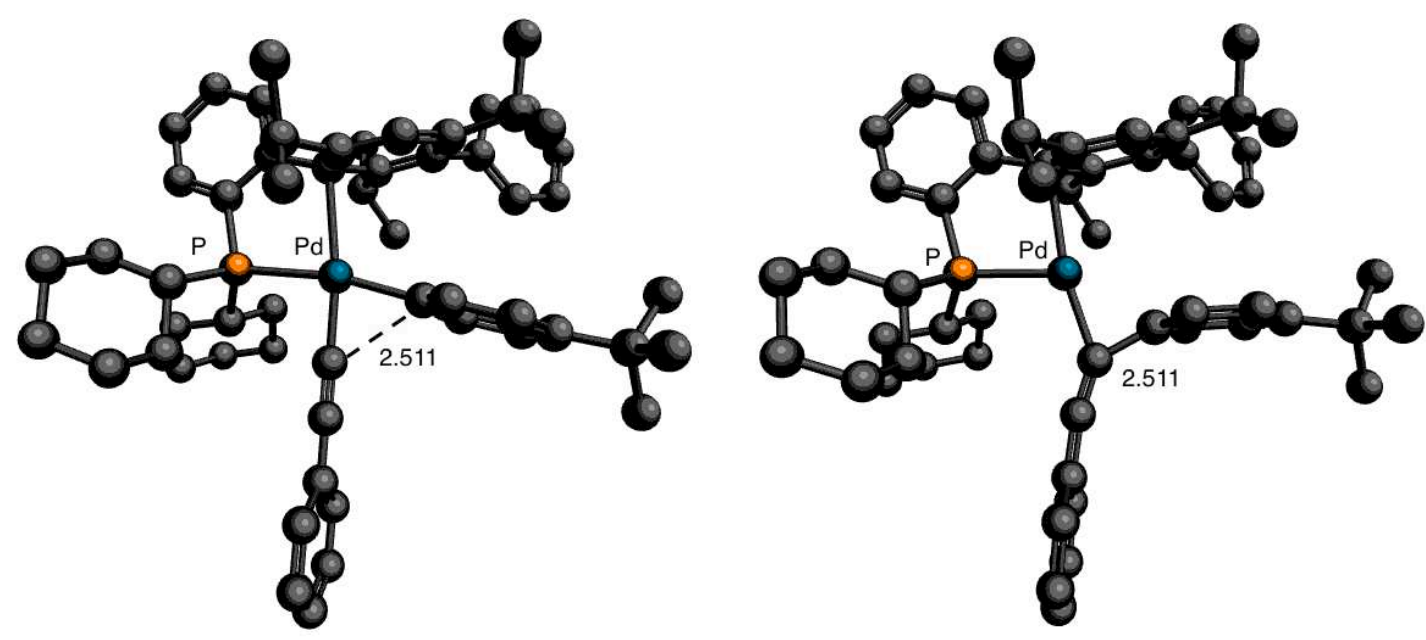

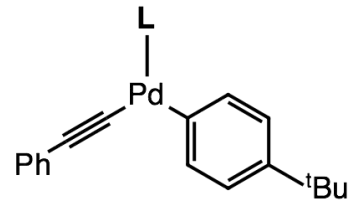

Reactant complex

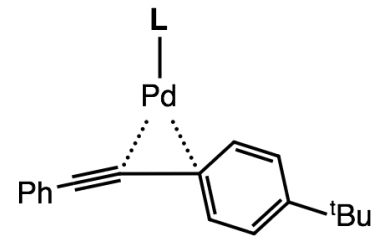

Product complex

Figure S14. Geometric structure of TS4 (top), and structures corresponding to the reactant complex (4) and products $(\mathbf{A}+\mathbf{P 1})$ on the intrinsic reaction path for reductive elimination. Interatomic distances are given in Ångstroms. 
TS-S2A

Energy $=-611.212296$ a.u.

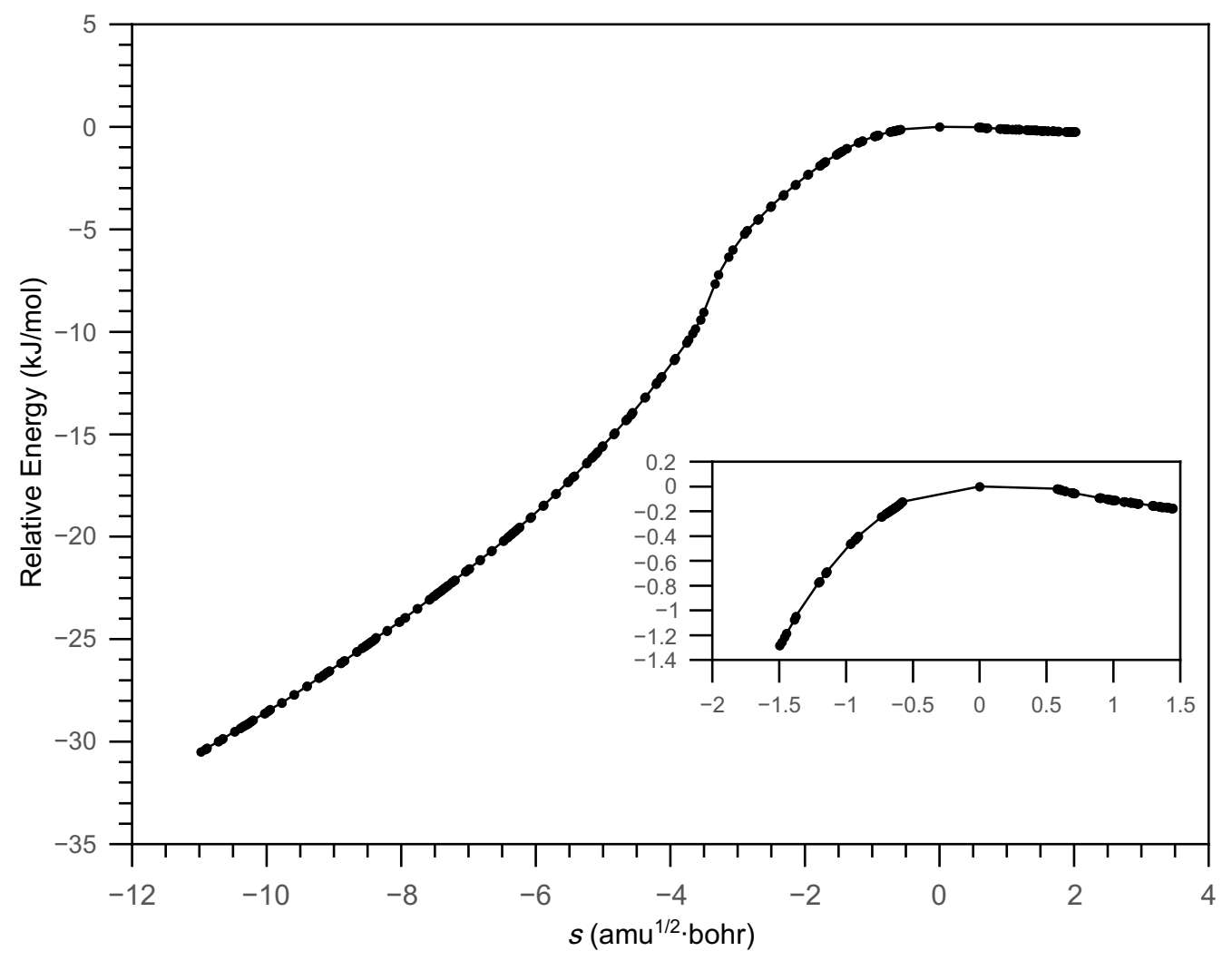

Figure S15. Reaction path following for transition state TS-S2, corresponding to the acid-base reaction $\mathbf{S} 2$ with $\mathrm{Cs}_{2} \mathrm{CO}_{3}$ to form $\mathbf{S 2 A}$. The inset shows the magnified intrinsic reaction path profile from $s=-1.5$ to $s=1.5$. 


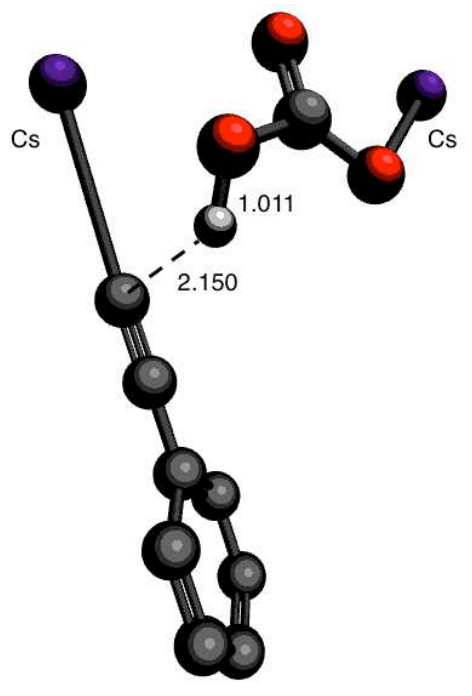

TS-S2
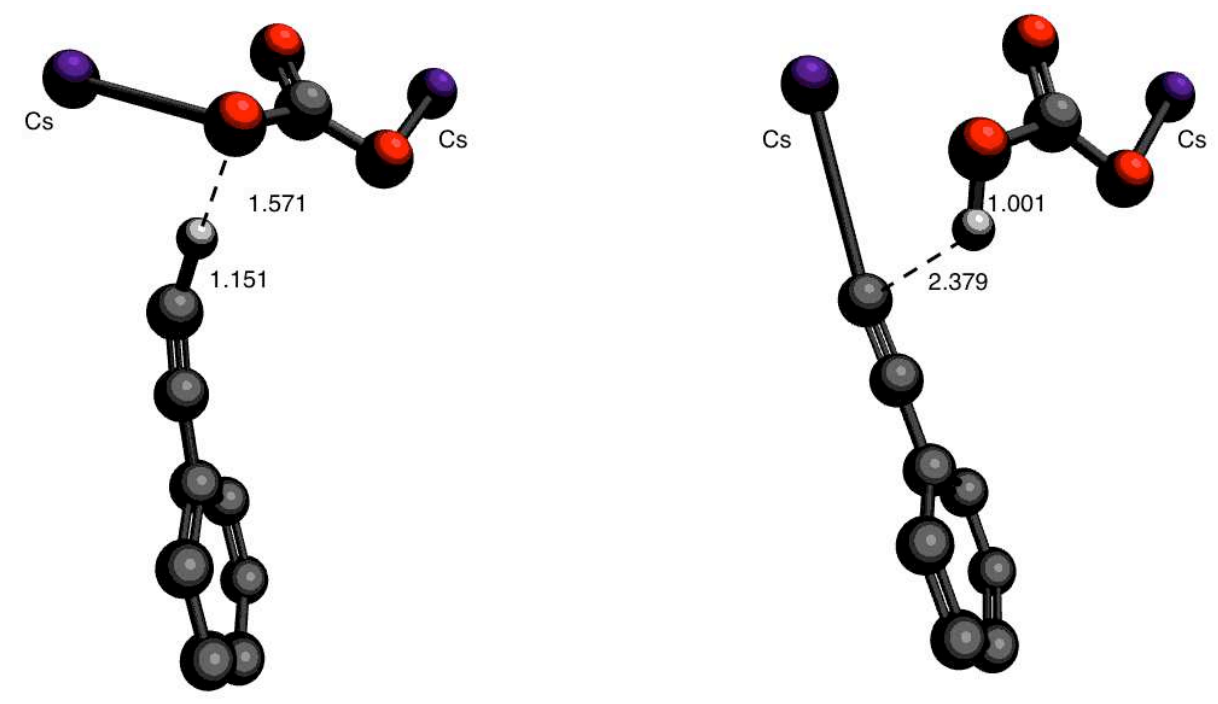

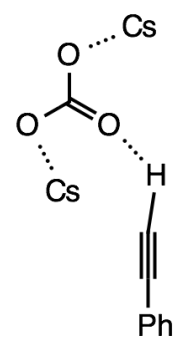

Reactant complex

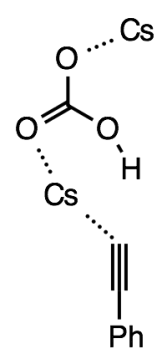

Product complex

Figure S16. Geometric structure of TS-S2 (top), and structures corresponding to the reactant complex, $\mathbf{S 2}+\mathrm{Cs}_{2} \mathrm{CO}_{3}$, and product $\mathbf{S 2 A}$ on the intrinsic reaction path for $\mathbf{S} 2+\mathrm{Cs}_{2} \mathrm{CO}_{3}$ reaction. Interatomic distances are given in Ångstroms. 
TS-D1

Energy = -2052.204556 a.u.

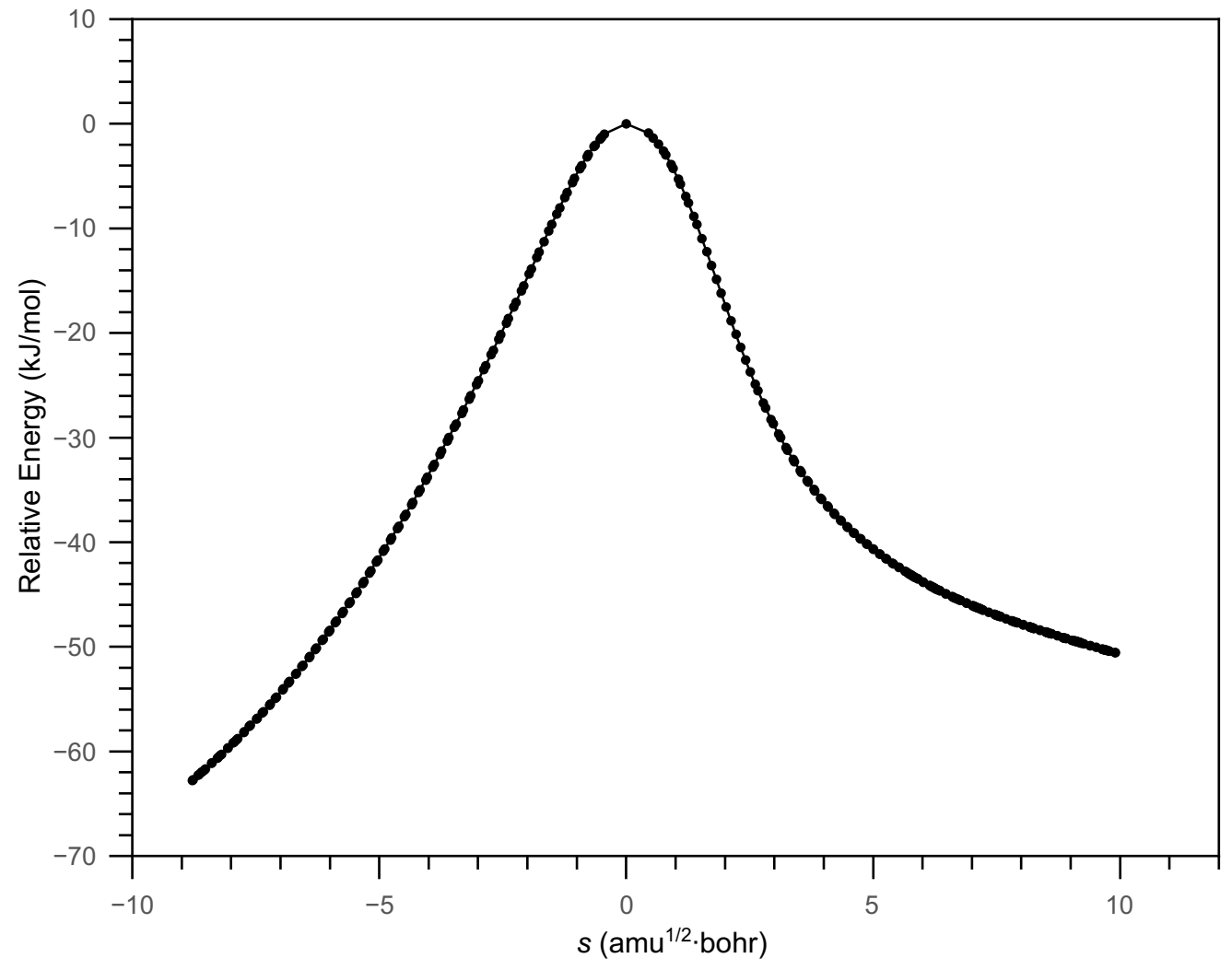

Figure S17. Reaction path following for transition state TS-D1, corresponding to the dearomative rearrangement of 2 to form D1. 


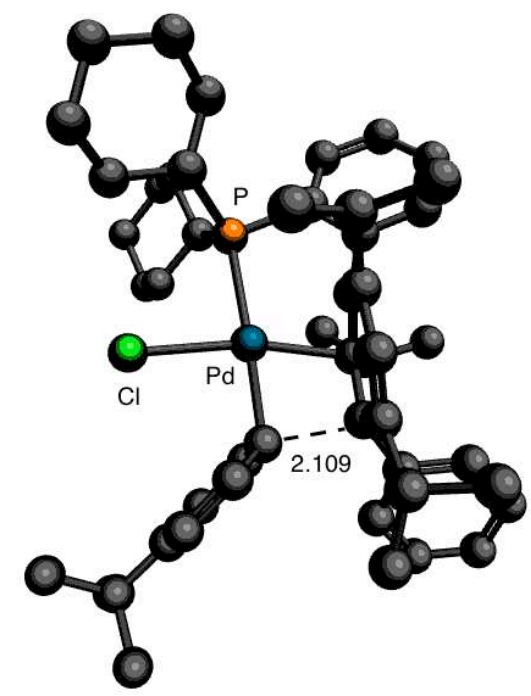

TS-D1

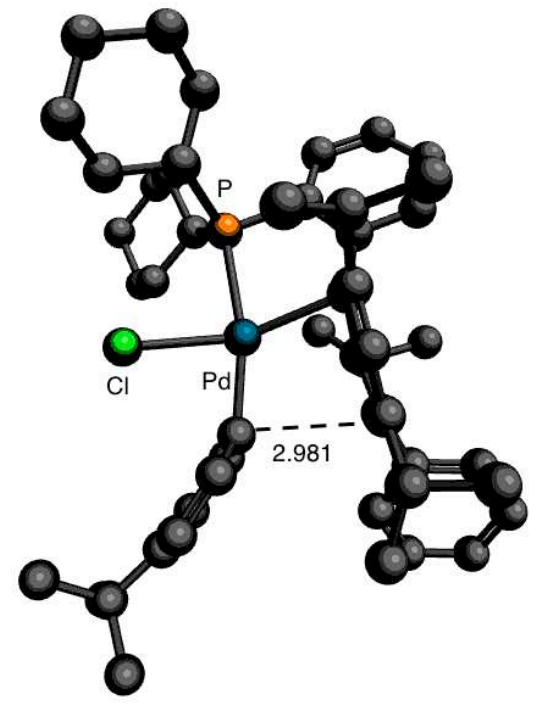

Reactant

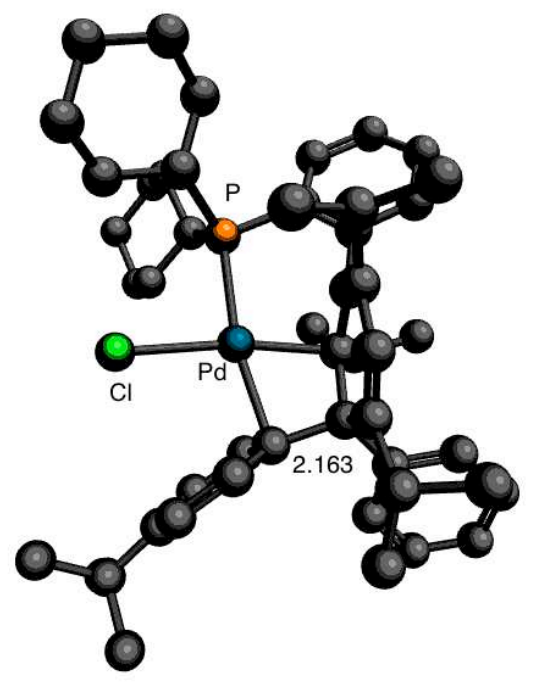

Product

Figure S18. Geometric structure of TS-D1 (top), and structures corresponding to the reactant $\mathbf{2}$, and product D1 on the intrinsic reaction path for dearomative rearrangement (DR1 pathway). Interatomic distances are given in Ångstroms. 
TS-D2

Energy $=-2052.228225$ a.u.

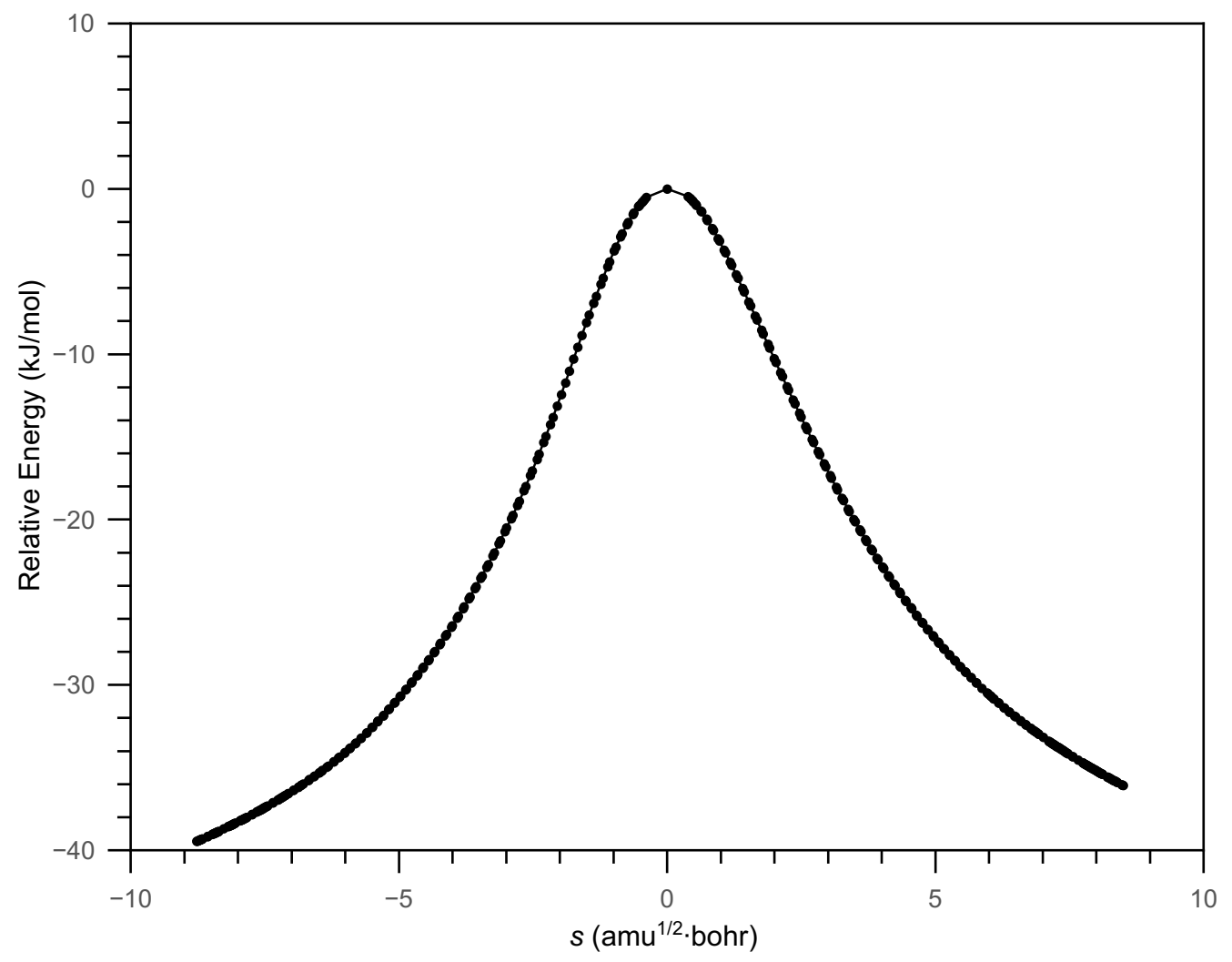

Figure S19. Reaction path following for transition state TS-D2, corresponding to the dearomative rearrangement of $\mathbf{2}$ to form $\mathbf{D} 2$. 


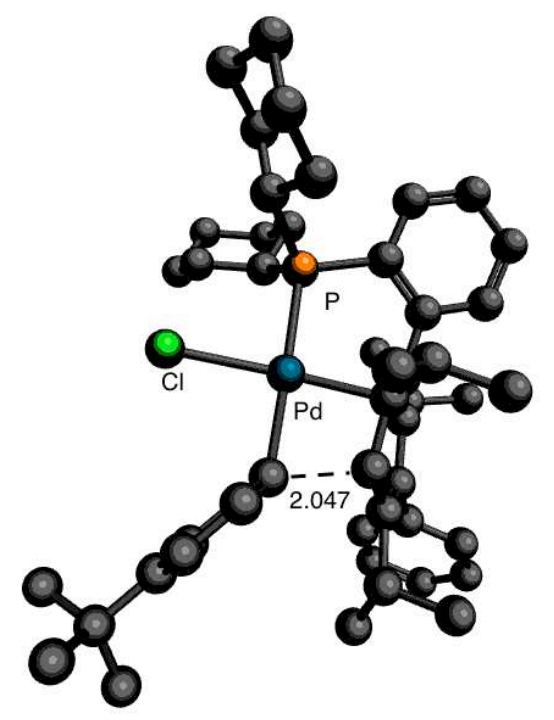

TS-D2

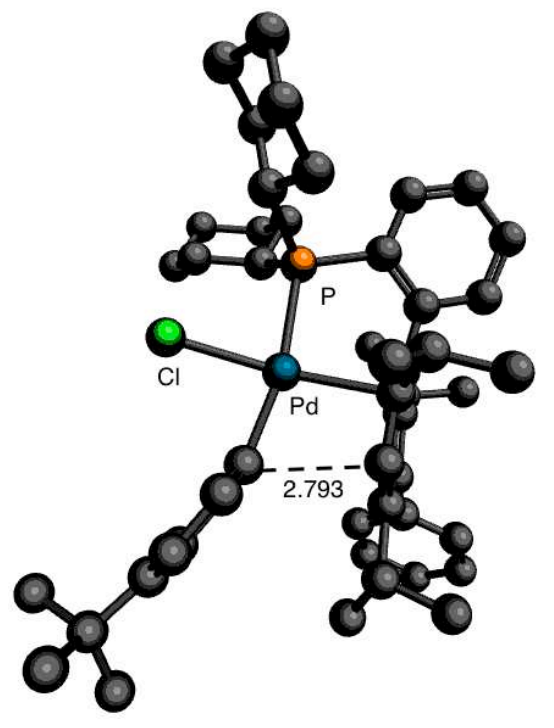

Reactant

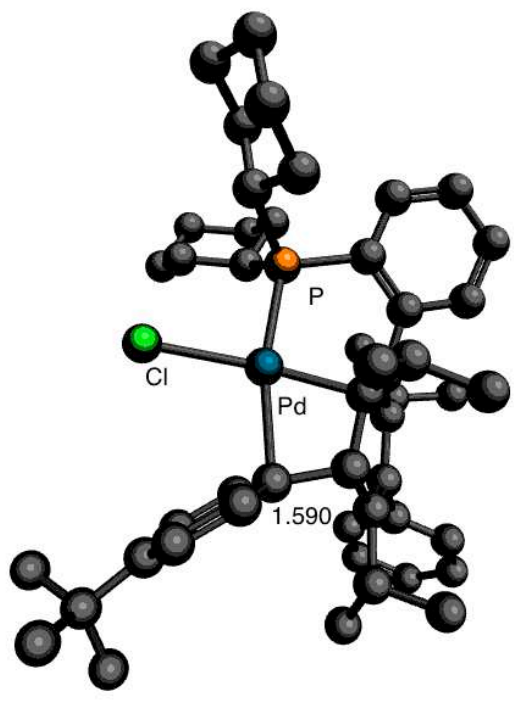

Product

Figure S20. Geometric structure of TS-D2 (top), and structures corresponding to the reactant 2, and product D2 on the intrinsic reaction path for dearomative rearrangement (DR1 pathway). Interatomic distances are given in Ångstroms. 
b. IRC analyses for the rate-determining step, $\mathbf{2} \rightarrow[$ TS2] $\rightarrow \mathbf{3}$ for $\mathrm{R}=\mathrm{H}, \mathrm{Me}$, OMe, F, CN are shown below.

$\mathrm{R}=\mathrm{H}$

Energy $=-2432.852169$ a.u.

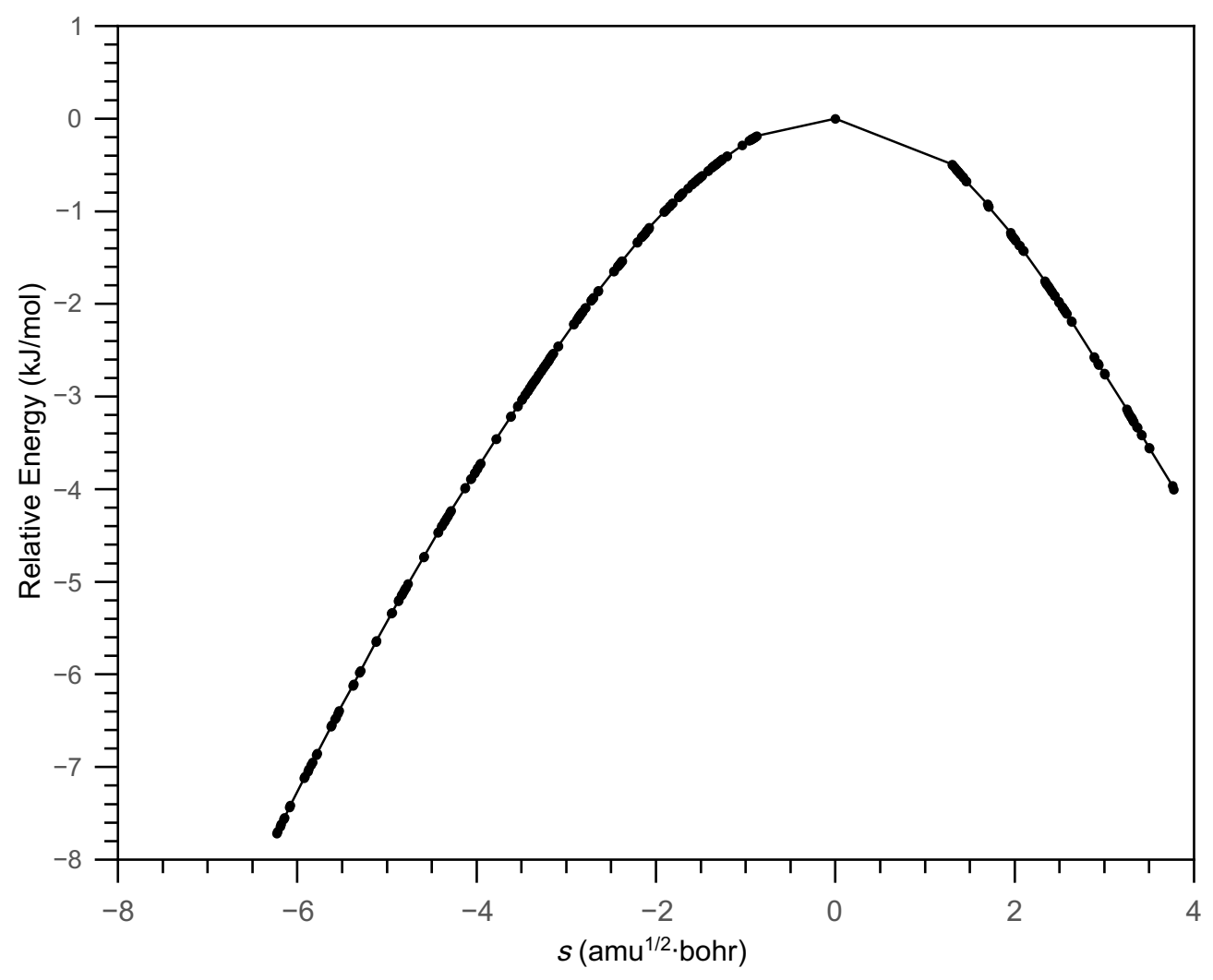

Figure S21. Reaction path following for transition state TS2 for $\mathrm{R}=\mathrm{H}$, corresponding to the direct addition of alkyne $\mathbf{S} 2$ to the oxidative addition product 2 to yield 3 . 


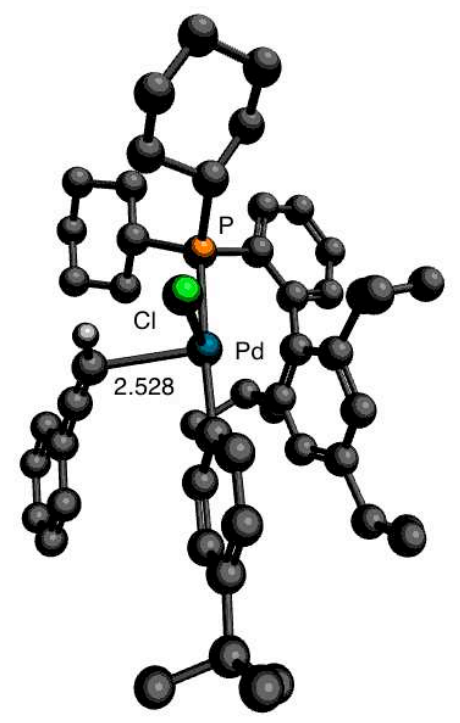

TS2
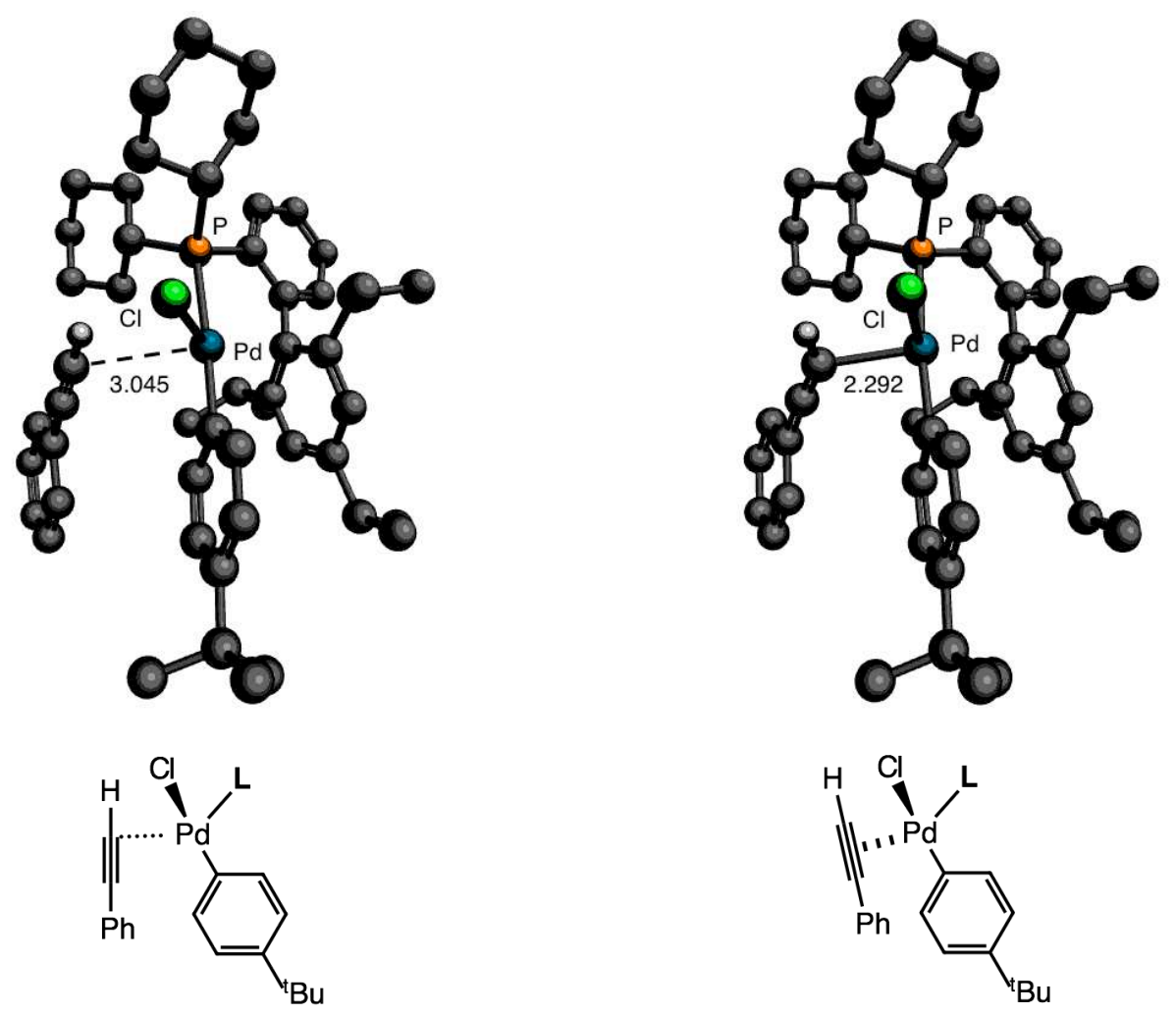

Reactant complex

Product complex

Figure S22. Geometric structure of TS2 (top), and structures corresponding to the reactant $(2+\mathbf{S} 2)$ and product (3) complexes on the intrinsic reaction path for direct alkyne addition for $\mathrm{R}=\mathrm{H}$. Interatomic distances are given in Ångstroms. 
$\mathrm{R}=\mathrm{Me}$

Energy $=-2168.722615$ a.u.

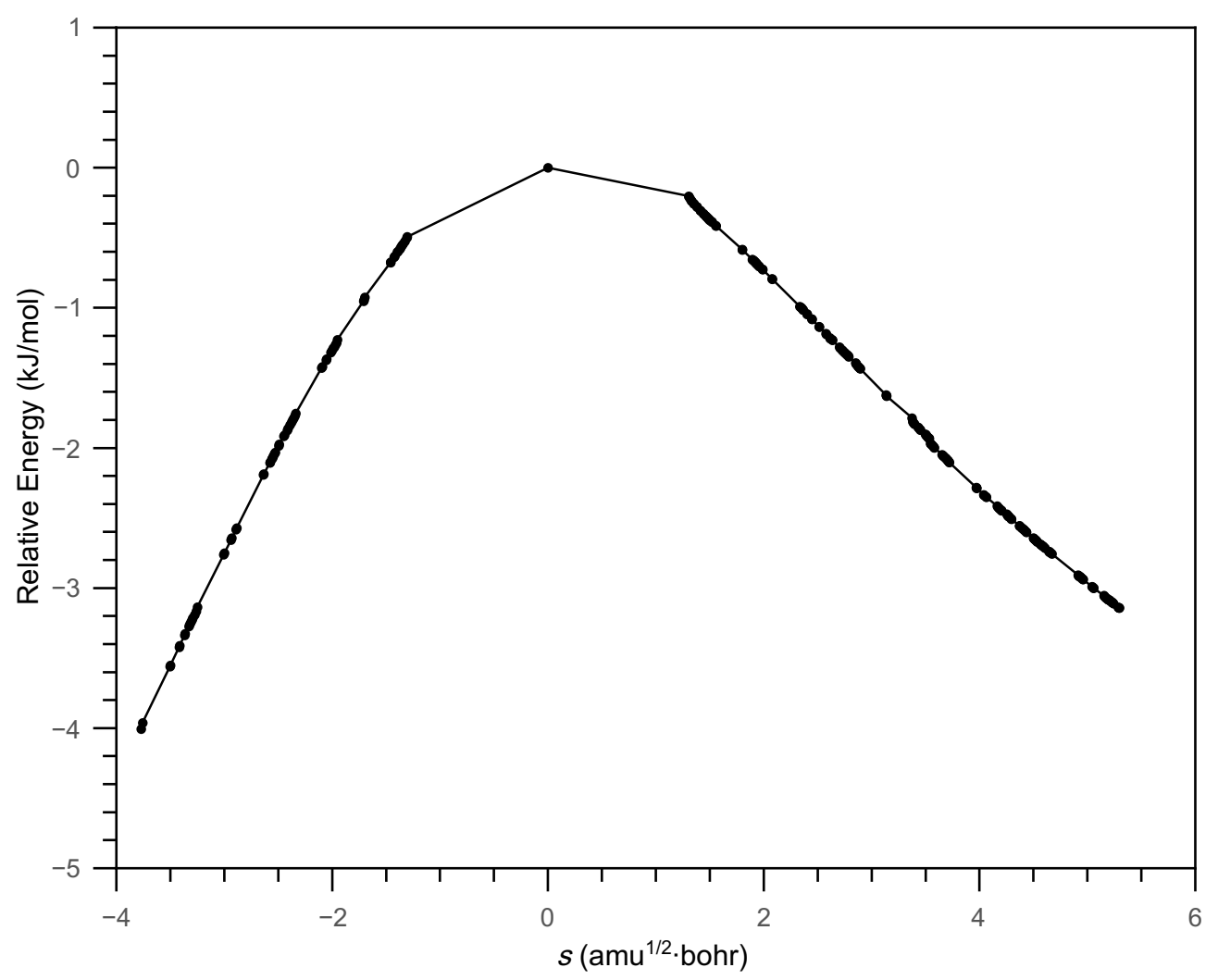

Figure S23. Reaction path following for transition state TS2 for R=Me, corresponding to the direct addition of alkyne $\mathbf{S} 2$ to the oxidative addition product $\mathbf{2}$ to yield $\mathbf{3}$. 


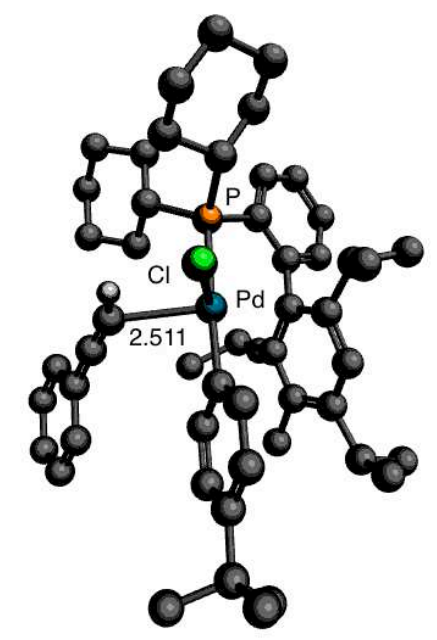

TS2
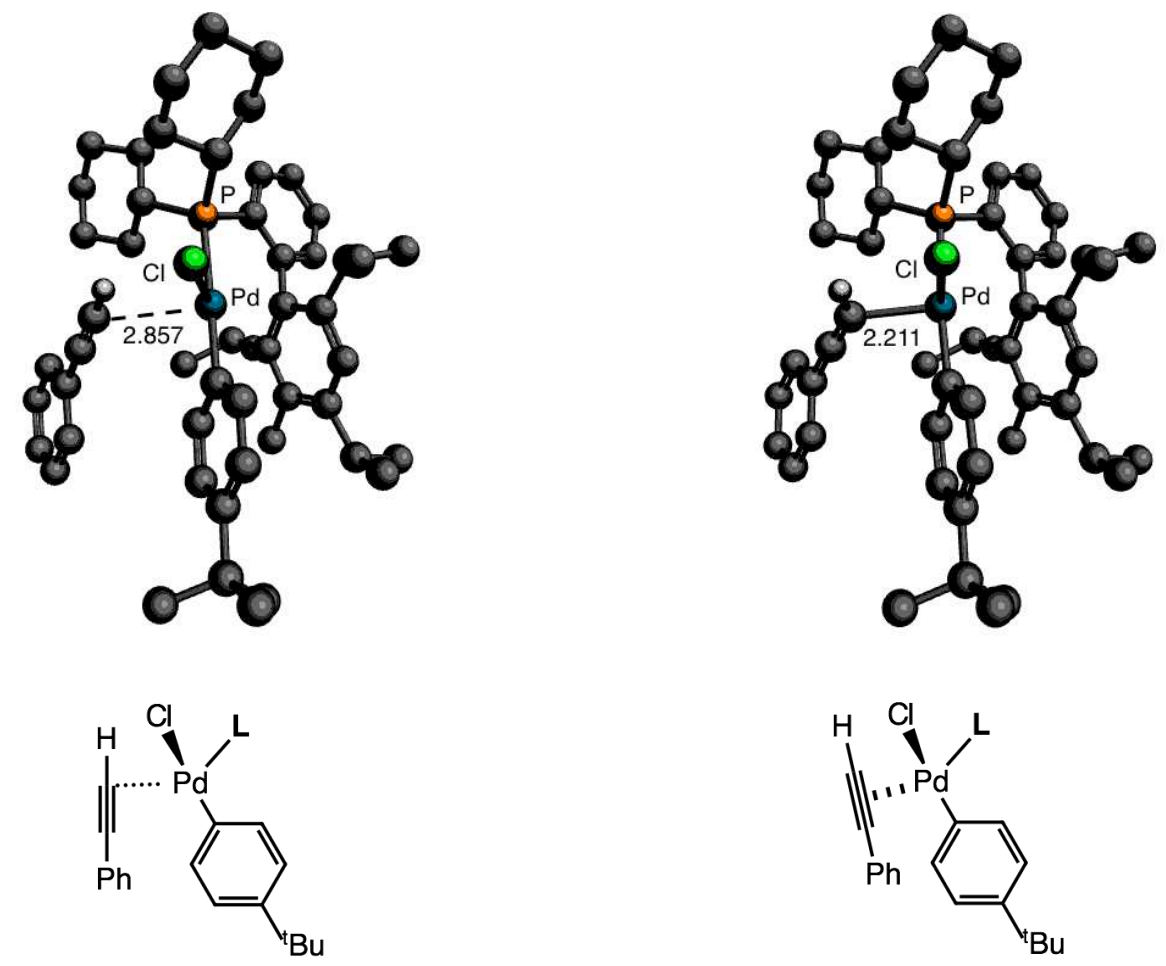

Reactant complex

Product complex

Figure S24. Geometric structure of TS2 (top), and structures corresponding to the reactant $(2+\mathbf{S 2})$ and product (3) complexes on the intrinsic reaction path for direct alkyne addition for $\mathrm{R}=\mathrm{Me}$. Interatomic distances are given in Ångstroms. 
$\mathrm{R}=\mathrm{OMe}$

Energy $=-2243.828518$ a.u.

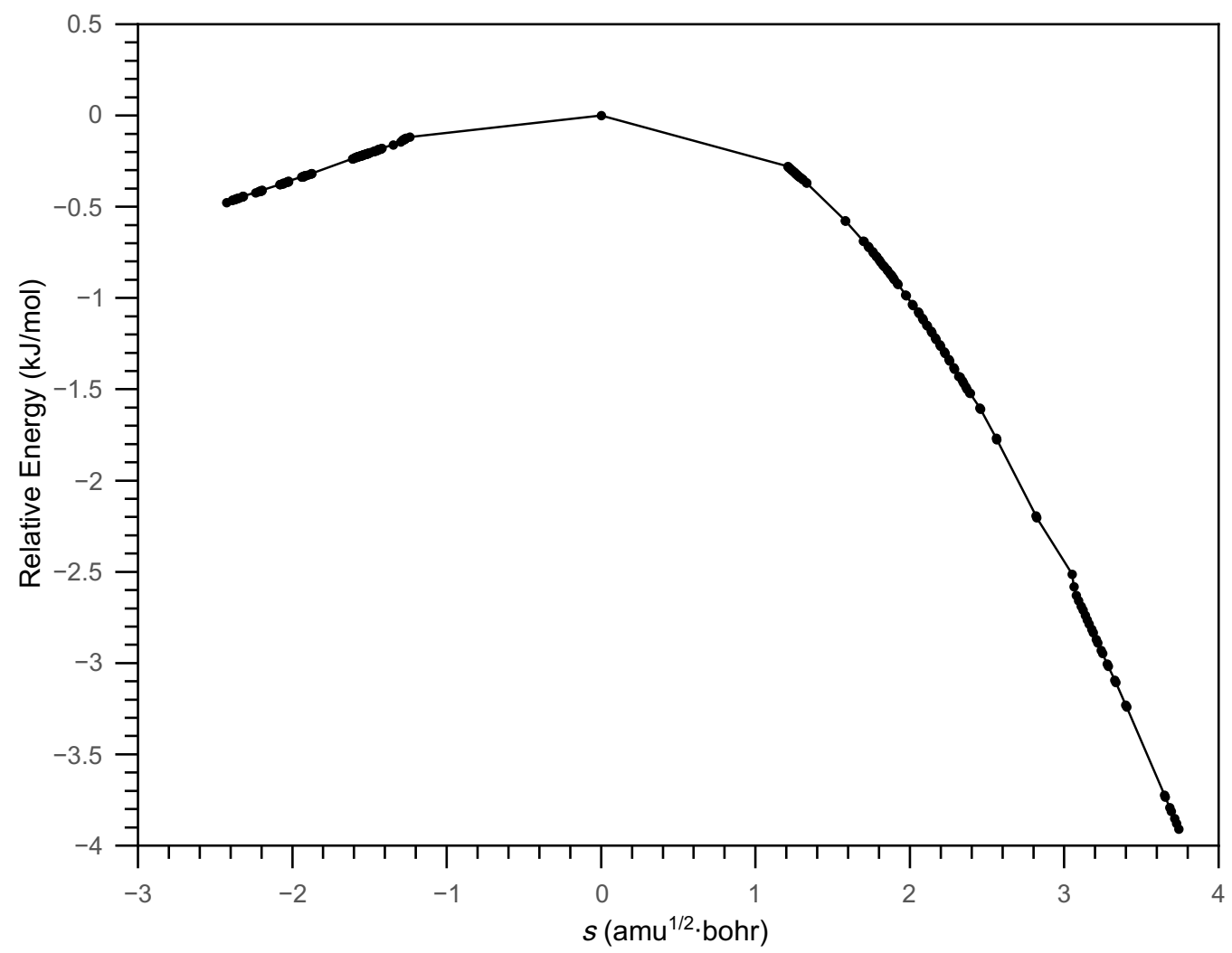

Figure S25. Reaction path following for transition state TS2 for R=OMe, corresponding to the direct addition of alkyne $\mathbf{S} 2$ to the oxidative addition product 2 to yield 3 . 


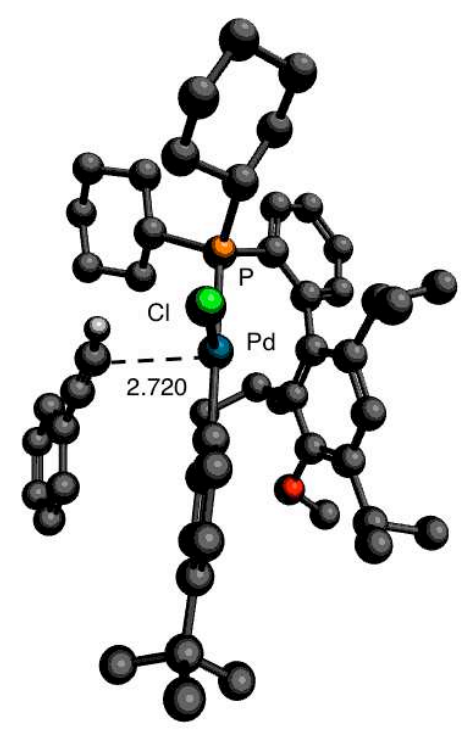

TS2
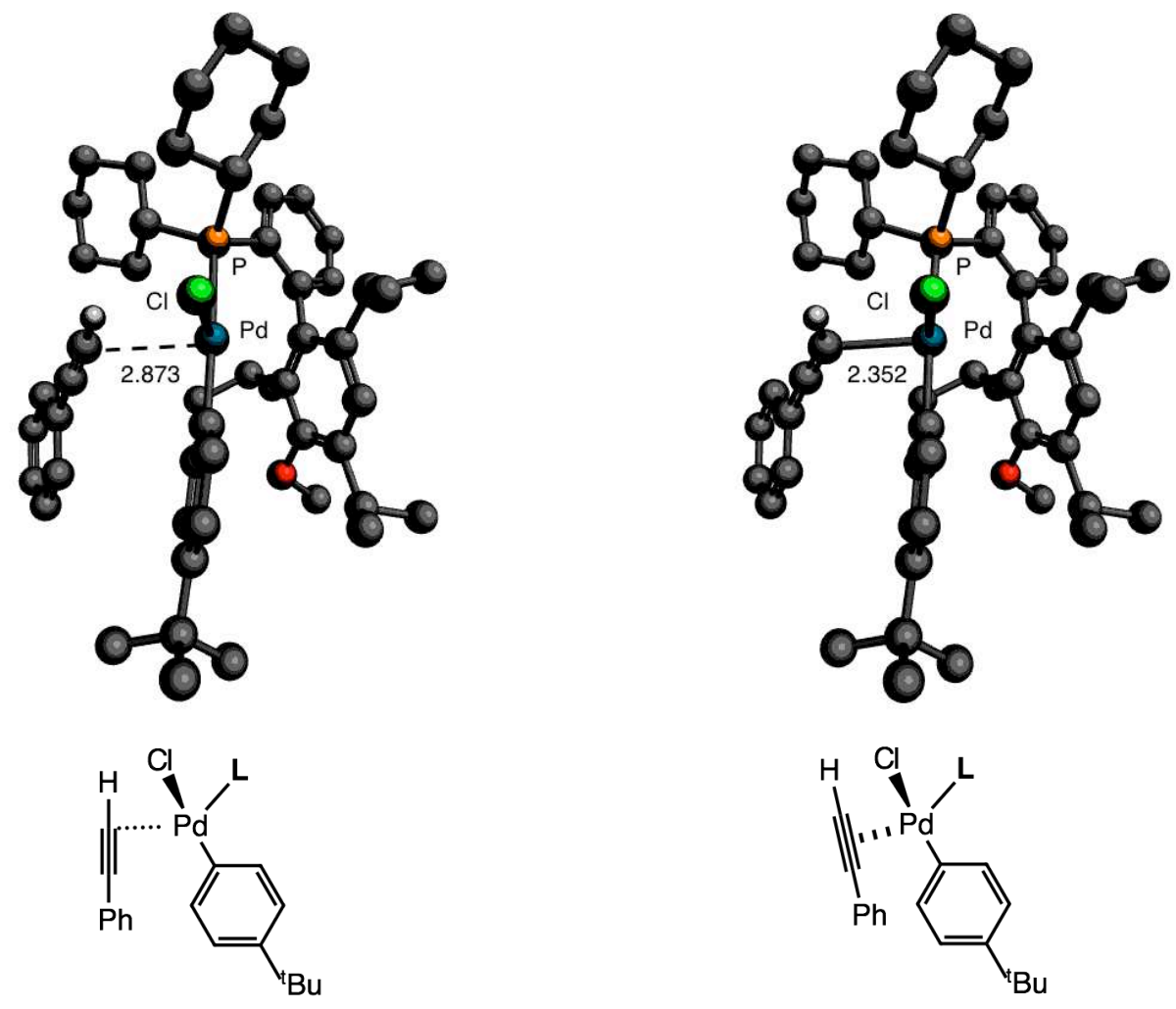

Reactant complex

Product complex

Figure S26. Geometric structure of TS2 (top), and structures corresponding to the reactant $(2+\mathbf{S} 2)$ and product (3) complexes on the intrinsic reaction path for direct alkyne addition for $\mathrm{R}=\mathrm{Me}$. Interatomic distances are given in Ångstroms. 
$\mathrm{R}=\mathrm{F}$

Energy $=-2228.612934$ a.u.

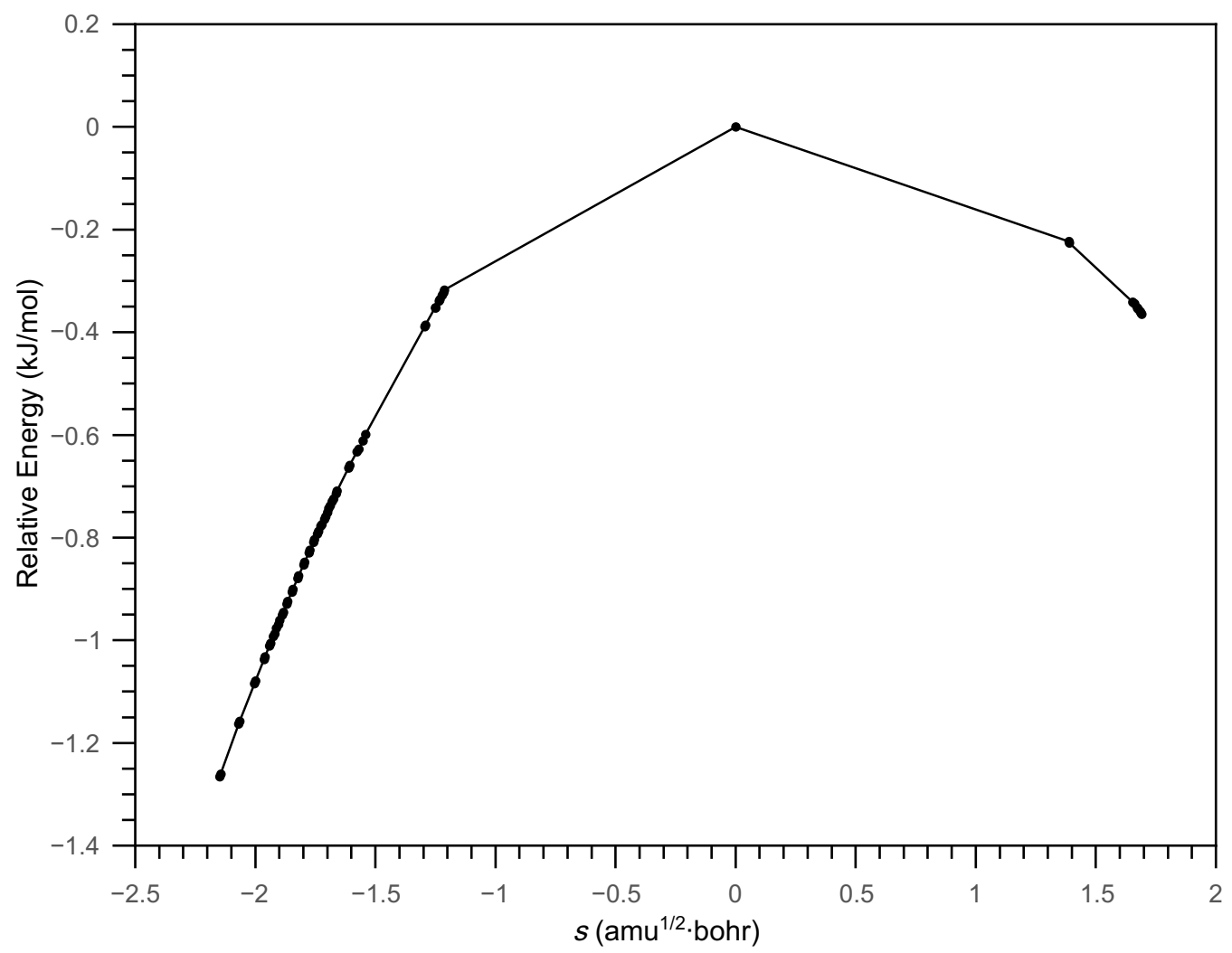

Figure S27. Reaction path following for transition state TS2 for $R=F$, corresponding to the direct addition of alkyne $\mathbf{S} 2$ to the oxidative addition product 2 to yield 3. 


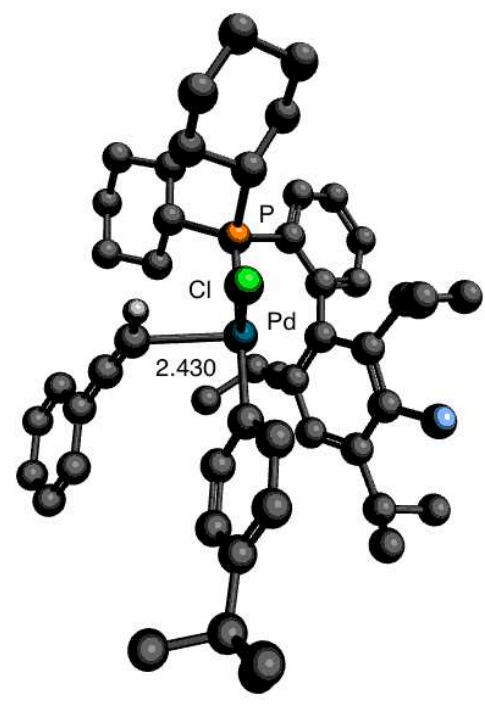

TS2
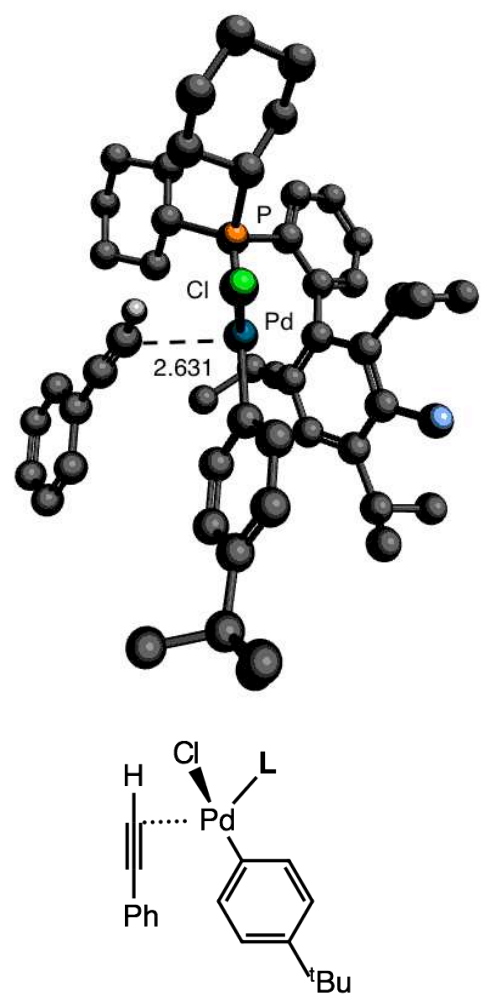

Reactant complex
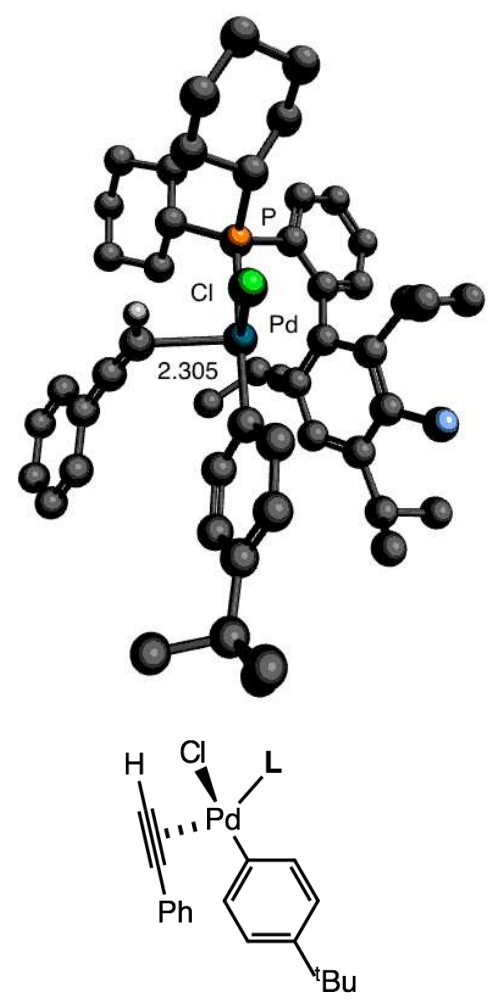

Product complex

Figure S28. Geometric structure of TS2 (top), and structures corresponding to the reactant $(2+\mathbf{S} 2)$ and product (3) complexes on the intrinsic reaction path for direct alkyne addition for $\mathrm{R}=\mathrm{F}$. Interatomic distances are given in Ångstroms. 
$\mathrm{R}=\mathrm{CN}$

Energy = -2221.598910 a.u.

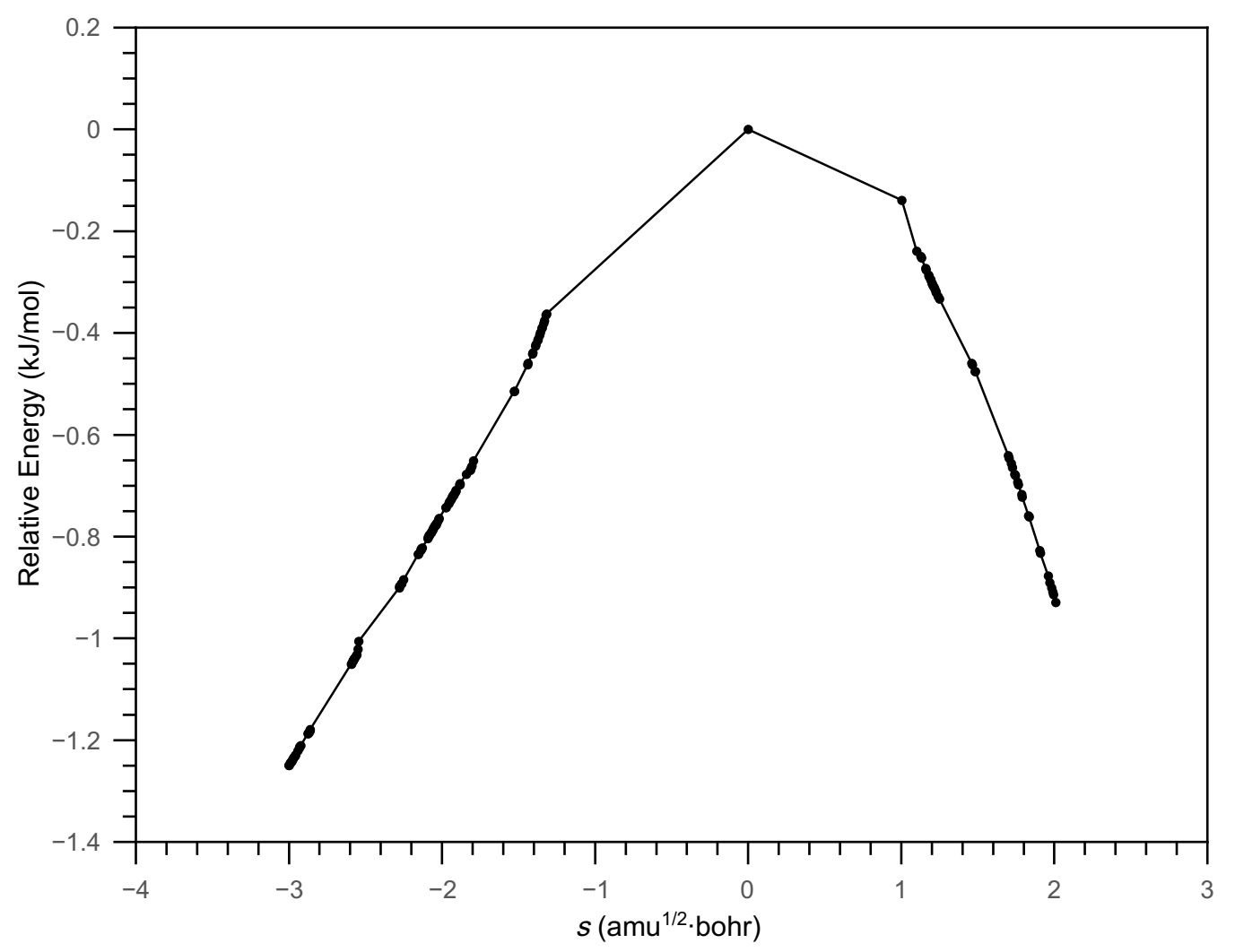

Figure S29. Reaction path following for transition state TS2 for $\mathrm{R}=\mathrm{CN}$, corresponding to the direct addition of alkyne $\mathbf{S} 2$ to the oxidative addition product 2 to yield 3. 


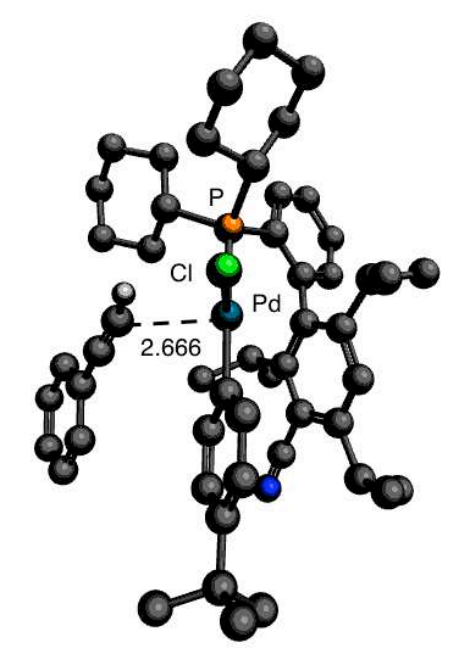

TS2
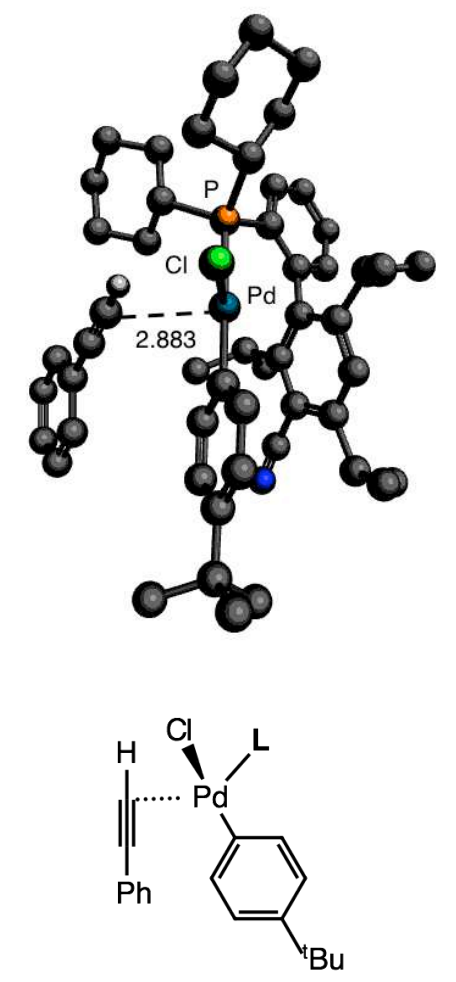

Reactant complex
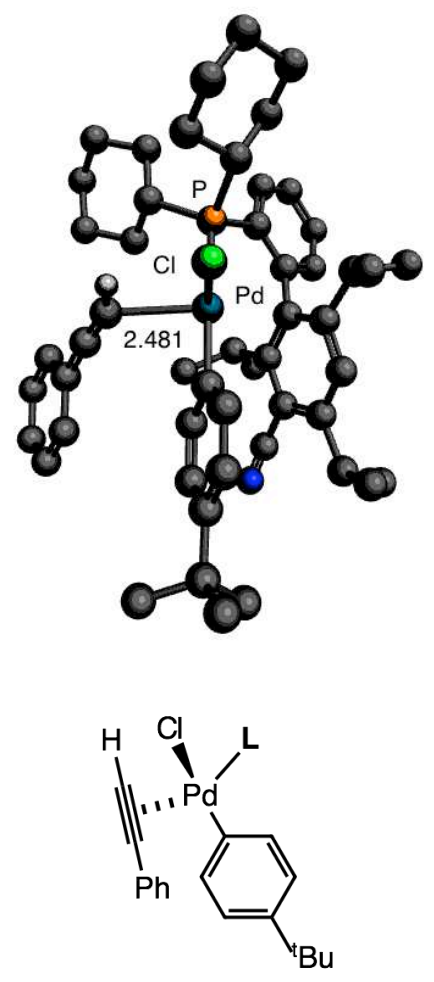

Product complex

Figure S30. Geometric structure of TS2 (top), and structures corresponding to the reactant $(2+\mathbf{S} 2)$ and product (3) complexes on the intrinsic reaction path for direct alkyne addition for $\mathrm{R}=\mathrm{CN}$. Interatomic distances are given in Ångstroms. 